\title{
Large-Scale Studies on Slat Noise Reduction
}

\author{
M. Herr*, M. Pott-Pollenske, R. Ewert $†$ D. Boenke, J. Siebert, J. Delfs $\ddagger$ A. Rudenko ${ }^{\S}$ \\ German Aerospace Center (DLR), Lilienthalplatz 7, D-38108 Braunschweig, Germany
}

\author{
A. Büscher, H. Friedel!, and \\ Airbus Operations GmbH, Airbus-Allee 1, D-28199 Bremen, Germany \\ I. Mariotti** \\ Airbus Operations S.A.S, 316 Route de Bayonne, F-31060 Toulouse, Cedex 9, France
}

\begin{abstract}
Slat noise research activity within the EC co-financed project OPENAIR involved both experimental and numerical studies at the new large (1:3.3-scaled) swept high-lift wing model F15LS. Experiments were performed in the DNW-LLF (Large Low-speed Facility) to verify the noise reduction benefit of selected slat noise reduction concepts under more realistic test conditions than in precursor projects. Moreover, the gained test data served to extend current slat noise validation datasets towards larger Reynolds numbers, i. e. up to $R e=5.1 \times 10^{6}$ in the current experiment. Slat noise reduction concepts under review were 1) slat gap/overlap setting variations, and 2) an adaptive slat with the potential to reduce the gap width for noise reduction. Both concepts were proven highly efficient: Sealing of the gap leads to a maximum 5-dB noise reduction at wing level, equivalent to a full elimination of the slat noise source. Optimized slat settings or an adaptive slat with partially closed gap are suited to reduce slat noise by about $2-3 \mathrm{~dB}$ at wing level while producing negligible aerodynamic impact at the operative test angles of attack within the linear polar region. When transposing these results to overall aircraft flight conditions, optimized slat settings might bring about a 0.5-EPNdB reduction of approach certification noise levels, provided all other relevant noise sources than the slat remain untreated. CAA (Computational Aeroacoustics) prediction results derived with DLR's PIANO and DISCO codes coupled with RANS-based stochastic source models revealed a generally good reproduction of the measured trends.
\end{abstract}

\section{Nomenclature}

Parameter definition and units

$\begin{array}{lll}C_{L_{\max }} & - & \text { maximum lift coefficient } \\ c_{p} & - & \text { static pressure coefficient } \\ c_{n} & - & \text { normal force coefficient } \\ f & 1 / \mathrm{s} & \text { (narrowband) frequency } \\ f_{c} & 1 / \mathrm{s} & 1 / 3 \text {-octave band center frequency } \\ L_{p}, L_{p(1 / 3)} & \mathrm{dB} & \text { narrowband or } 1 / 3 \text {-octave band sound pressure level, referenced to } \\ & & \text { a unit span and a unit observer distance }\end{array}$

*Research Engineers, DLR Institute of Aerodynamics and Flow Technology, Technical Acoustics, michaela.herr@dlr.de, michael.pott-pollenske@dlr.de, AIAA Members, dirk.boenke@dlr.de.

${ }^{\dagger}$ Senior Scientist, DLR Institute of Aerodynamics and Flow Technology, Technical Acoustics, roland.ewert@dlr.de, AIAA Senior Member.

${ }^{\ddagger}$ Head of Technical Acoustics Department, DLR Institute of Aerodynamics and Flow Technology, jan.delfs@dlr.de, AIAA Senior Member.

§Research Engineer, DLR Institute of Composite Structures and Adaptive Systems, Adaptronics, anton.rudenko@dlr.de.

『Research Engineer, Department of Aerodynamic Design, alexander.buescher@airbus.com, AIAA Member.

" Research Engineer, Future Projects Office, hendrik.friedel@airbus.com.

**Research Engineer, Acoustics Department, irene.mariotti@airbus.com. 


$\begin{array}{lll}L_{\text {pnorm }}, L_{p(1 / 3) \text { norm }} & \mathrm{dB} & \text { normalized sound pressure levels, Eqs. (3) and (5) } \\ l_{s} & \mathrm{~m} & \text { slat chord length } \\ M_{\infty} & - & \text { time-averaged free stream Mach number } \\ R_{e} & - & \text { chord-based Reynolds number } \\ S t & - & \text { Strouhal number, Eqs. (4) and (6) } \\ u_{\infty} & \mathrm{m} / \mathrm{s} & \text { time-averaged free stream velocity } \\ \alpha & \circ & \text { aerodynamic angle of attack (real flight, 2D) } \\ \alpha_{W T} & \circ & \text { geometric (wind tunnel) angle of attack } \\ \epsilon_{\max } & - & \text { maximum strain rate } \\ \theta & \circ & \text { polar observation angle }\end{array}$

\section{Introduction}

$\mathrm{T}$ HE EC co-financed project OPENAIR (Optimization for low environmental noise impact aircraft) aims to increase the technology readiness of selected - new and already proven - aircraft noise reduction technologies. The current report concentrates on the slat noise research activity within work package WP4.2 (Wing Systems) with the following research objectives:

- extension of current slat noise validation data bases towards more realistic test conditions (larger scale, swept wing) than in precursor projects to support the further development of DLR's numerical slat noise prediction capability for aeroacoustic design;

- verification of the noise reduction benefit of already proven slat noise reduction concepts for these extended test conditions.

Slat noise reduction concepts that have been revisited and further developed were:

- optimized slat gap/overlap settings, ${ }^{13,21}$

- adaptive slat - as originally proposed by Delfs, ${ }^{6}$ later filed for patent ${ }^{19}$ - that actively reduces the gap width by trailing-edge shape deformation to reduce noise under typical approach conditions but restores the original gap width / maximum lift if necessary.

\section{I.A. Optimized slat gap/overlap settings}

OPENAIR slat noise studies build upon the outcome of the forerunner EC-project TIMPAN, where the noise differences attributed to slat setting variations were numerically predicted and experimentally validated at a fourfold smaller 1:13.2-scaled 2D high-lift system (F16 model) in DLR's Acoustic Wind Tunnel Braunschweig (AWB). ${ }^{13}$ In both TIMPAN and OPENAIR DLR's CAA code PIANO/RPM was applied for the numerical slat noise predictions, while numerical studies within OPENAIR covered also the further-development of the PIANO/FRPM- and DISCO/FRPM frameworks (see section IV). In figure 1 the selected OPENAIR gap/overlap settings SO1-SO4 are referenced to these former (small Re) 2D CAA prediction results from TIMPAN. These predictions were used to identify optimized configurations based on estimates of

- the noise reduction potential $(\Delta \mathrm{SPL}$ in the figure denote overall sound pressure level differences compared to the TIMPAN reference setting in terms of integrated values along a ground measurement line), and

- the aerodynamic impact (detrimental effects on maximum lift coefficients $C_{L_{\max }}$ ).

As a result of this first assessment, assisted by the Airbus Technology Evaluator ${ }^{4}$ process, optimal solutions were expected in the gap/overlap range marked by the red rectangle in the figure. A suitable aeroacoustic cost function for slat setting optimization has been for example reported by Delfs. ${ }^{5}$ 
TIMPAN settings Pos $1 \ldots$
$\triangle$ OPENAIR settings SO $1 \ldots$
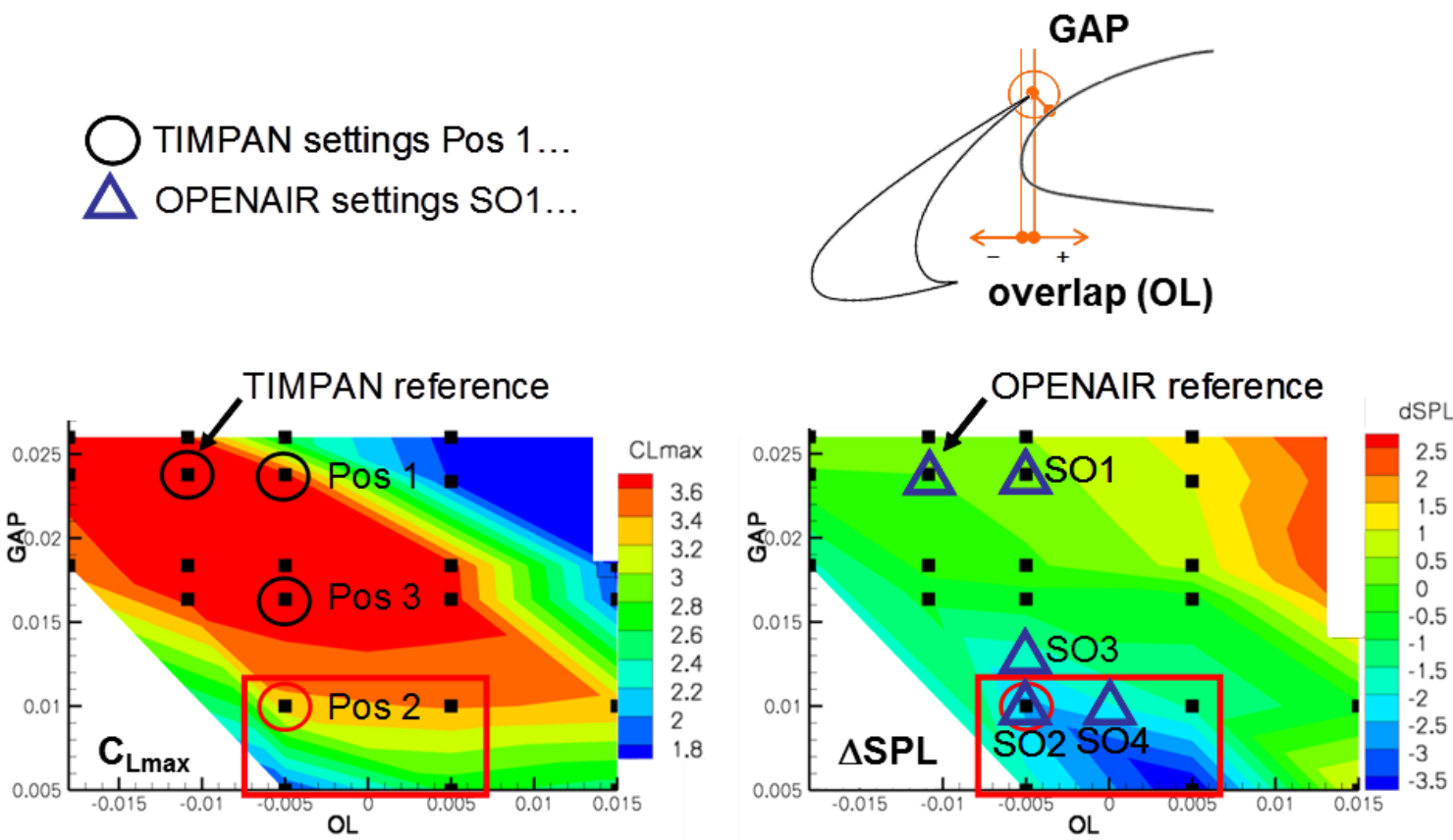

Figure 1. TIMPAN CFD/CAA simulation data ${ }^{13}$ revisited; survey on (left) TIMPAN slat settings Pos $1-3$ and (right) OPENAIR settings SO1-SO4. TIMPAN simulation data refer to $30^{\circ}$ flap angle, $30^{\circ}$ slat angle, and $6^{\circ}$ angle-of-attack. The quietest TIMPAN setting, identical with SO2, is marked by the red circle.

\section{I.B. Adaptive slat concept}

The principal aerodynamic and acoustic behavior of the concept is illustrated in figure 2. Accordingly, the tailored solution will provide (1) a closed gap for noise reduction and sufficient aerodynamic performance under typical operation conditions and (2) the original gap/overlap setting and $C_{L_{\max }}$ of the conventional slat of the original aircraft configuration if needed (e. g. in cases of emergency, for certification). The following design requirements were defined for such an active slat device:

- system safety,

- retention of the original approach speed and thus $C_{L_{\max }}$ of the conventional slat with open gap,

- fast movement from the low-performance- to the high-performance position,

- limited weight and complexity impact,

- limited adverse influence on the flow (i. e. no tubes, etc. in the slat cove).

In particular, the following requirements were deduced:

- extension time of order $<2 \mathrm{~s}$,

- provision of a fail-save emergency release concept (inactive system = original gap),

- system reliability for all possible load conditions,

- prevention of failure cases where the system jams in the morphed position.

Note that an analogue certification procedure has been already applied to the Airbus A318 operated at London City Airport. Spoilers are deployed to allow for the required $5.5^{\circ}$ steep approach glide path but provide a passive retraction mechanism (assisted by the flow) to preserve the original $C_{L_{\max }}$ if needed. 

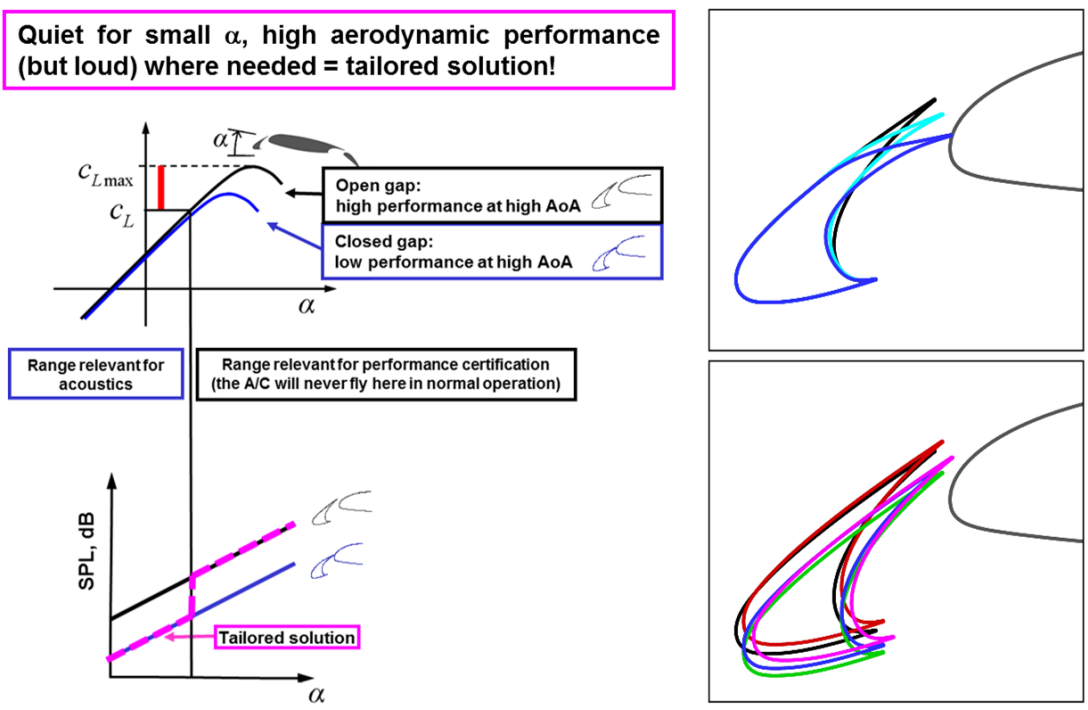

Figure 2. Left: $C_{L_{\max }}$-behavior of the adaptive slat concept and expected acoustic performance (schematic). Right: survey of OPENAIR test configurations.

\section{Selection of structurally feasible adaptive slat shapes}

2D iterative stress/strain FEM calculations served to specify technically feasible adaptive slat profile bending, accounting for conventional actuator specifications, feasible flexible skin materials and critical aerodynamic loads determined by the flight envelope. This preliminary assessment included the following aspects:

- morphing capability, i. e. identification of flexible structure topologies to achieve the required displacements and identification of materials that can cope with the relatively large strains resulting from morphing;

- material stiffness, i. e. quantification of unintended flow-induced deformations of the flexible skin for all critical cases of the aerodynamic loads envelope, the latter derived from supplementing, iterative CFD simulations;

- feasibility of actuator integration, i.e. allocation of installation space and power support, evaluation of system compatibility with the passive emergency release concept restoring the $C_{L_{\max }}$-behavior of the original high-lift wing.

Figure 3 illustrates the finally selected concept, consisting in a closed slat structure with a continuously bent outer shape and actuators located in the slat interior. The flexible skin could be realized by e. g. the flight-approved materials aluminum Al 7075 T6 or GFRP HexPly913. Example results from a strength analysis are shown in figure 3 (material failure criterion at $\epsilon_{\max }=1 \%$ strain). The displayed peaks at the force application points are rather the result of the simplified modeling of the actuator force (displacement defined on a single node row); these will reduce if a more realistic design for a distributed load insertion was applied. A more detailed 3D-structural and weight assessment of similar and alternative adaptive slat concepts is subject of currently ongoing work within follow-on project activities among the partners.

For the evaluation of the achievable noise benefit in the DNW-LLF the following two morphing steps were finally realized as solid model parts to replace the midspan region of the modular F15LS slat:

- Adaptive slat shape 1 provides an intermediate morphing step with the same gap/overlap settings as the conventional slat at setting SO3 (cf. figure 1).

- Adaptive slat shape 2 provides a fully closed gap with the expected maximum noise reduction potential. Such a configuration will not produce any slat noise in a dedicated slat noise CAA approach because the source is per se eliminated if the passage of the flow through the gap is prevented. The noise reduction potential of such a sealed gap configuration on wing level was quantified earlier as roughly $5-6 \mathrm{~dB}$ (results from mutual comparisons with a slatless high-lift system within the EC project NACRE). 
Figure 2 (right) shows the two selected shape variants along with the conventional slat settings tested in OPENAIR. For budgetary reasons these two exchangeable parts were lacking pressure taps required for a detailed aerodynamic assessment. Supplementary data for morphed slat $c_{p}$-distribution are expected from ongoing smaller-scaled tests.
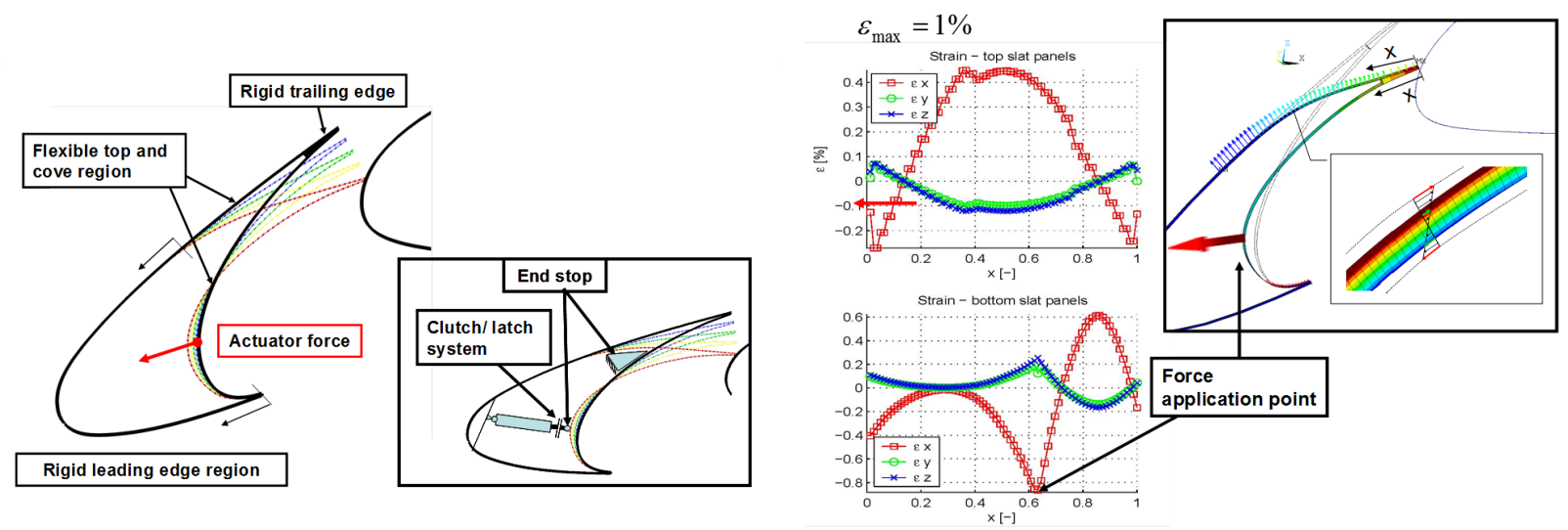

Figure 3. Left: selected adaptive slat concept. Insert: Proposed draft emergency releasing concept and end stops to avoid potential undesired shape deformation for the inactive system. Right: results from strength analysis for the GFRP material (3.2-3.75 $\mathrm{mm}$ thickness, 2-30 layers, quasi-isotropic layout).

\section{Experimental setup}

Figure 4 shows the DNW-LLF test setup with the F15LS high-lift system (constructed and manufactured within the German national project $\mathrm{FTEG}^{22}$ ) in its $2.5 \mathrm{D}$, i. e. $30^{\circ}$ swept configuration. The underlying geometry is a 2D projection of the 3D Airbus FNG wing section at a $51 \%$ spanwise station, hence supplementing the existing DLR F16, F15 model family (with $0.3 \mathrm{~m}$ and $0.6 \mathrm{~m}$ clean chord) by a corresponding large-scale (1.2-m clean chord, 7.2-m span) version. Slat and flap deployment angles of the F15LS high-lift system were set to $27.834^{\circ}$ and $25.0^{\circ}$, respectively. To provide an utmost constant spanwise lift distribution the model was mounted between two large (3-m-diameter) end plates with a vertical distance of $5 \mathrm{~m}$.

Model instrumentation comprised a total of 568 pressure taps and 12 unsteady surface pressure sensors in the slat cove region close to the trailing-edge. The distribution of tap positions with refinement along the midspan cross section has been documented by Pott-Pollenske et al. ${ }^{22}$ Noise measurements included array measurements (displaceable 140-microphone array pointing to the wing pressure side, cf. figure 4, left, frame in the background) and farfield single microphone measurements (3 rows of 12, 24 and 12 wall-mounted microphones according to figure 4, right). The interested reader might refer to Ref. 22 for further details.
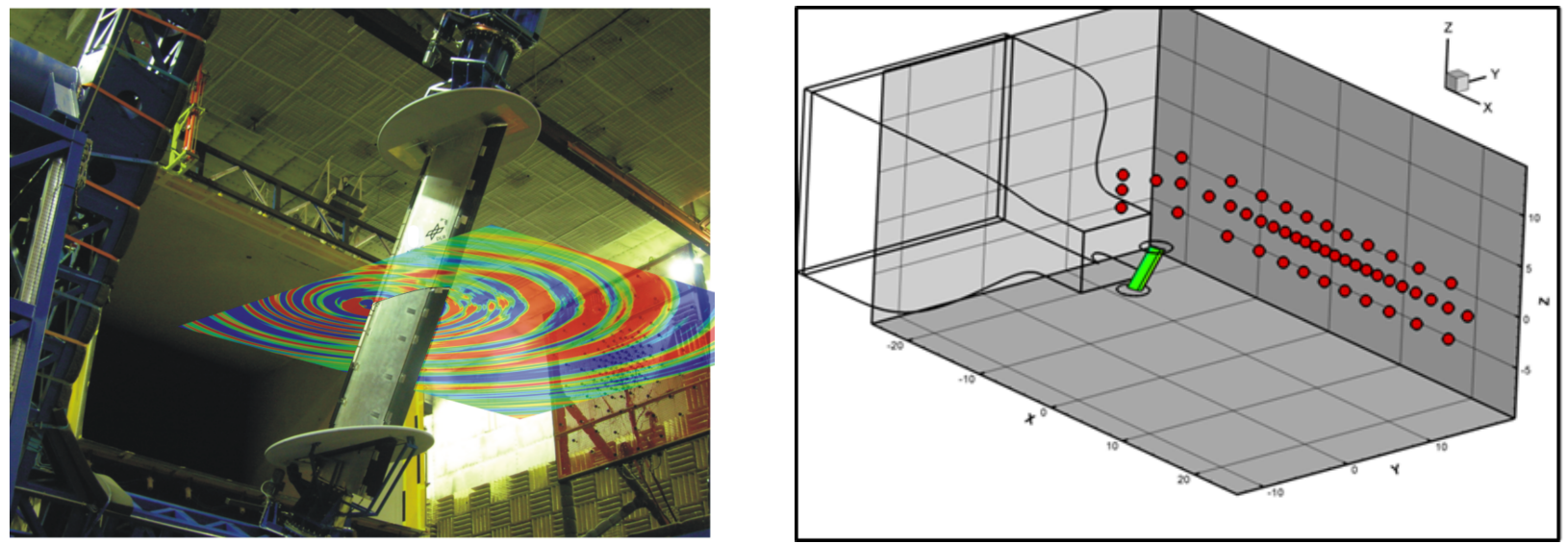

Figure 4. Left: F15LS installed in the DNW-LLF. Photographical composition with implanted snapshot of a PIANO/RPM slat noise simulation in a 2D-slice at midspan of the model. Right: used farfield microphone instrumentation. 


\section{III.A. Aerodynamic assessment - check of flow quality and definition of flow conditions}

A flow quality check at the beginning of the test proved that the targeted constant-lift conditions are actually available along a sufficiently large midspan region of the model, ${ }^{22}$ and no detrimental flow separation was identified (in particular at the flaps). Selection of the DNW-LLF test angles-of-attack was based on a CFDassisted comparison of target pressure distributions as follows: Ahead of the wind-tunnel campaign a target angle-of-attack of $\alpha=5^{\circ}$ (2D, real flight condition) was set, corresponding to $4.33^{\circ}$ for the swept $2.5 \mathrm{D}$ case. The corresponding target pressure distribution for the experiment was provided by a CFD computation in 2.5D to be realized at the center section of the WT model. Since the study particularly focuses on slat noise technology, the rear part of slat upper surface was selected as target region for the $c_{p}$-comparisons. Accordingly, the geometrical wind tunnel angle-of-attack $\alpha_{W T}=13^{\circ}$ was identified to well-represent the defined target. $^{22}$ Due to the described CFD-based comparison procedure no additional angle-of-attack correction was required; $\alpha_{W T}=10^{\circ} / 13^{\circ} / 16^{\circ}$ (corresponding to real flight $\alpha=3^{\circ} / 5^{\circ} / 7^{\circ}$ ) were selected for the systematic acoustic testing. Flow velocities $u_{\infty}$ were set to $45 \mathrm{~m} / \mathrm{s}, 55 \mathrm{~m} / \mathrm{s}$, and $65 \mathrm{~m} / \mathrm{s}$.

\section{III.B. Acoustic data acquisition, analysis and formatting}

To facilitate data reduction two different 'background noise (BGN) measurements' with different setups and hence, underlying objectives were conducted:

- Empty test section measurements served to extract the DNW-LLF background noise floor without the inherent effects of model and support. For farfield noise corrections, however, such BGN information is of limited utility because extraneous noise sources at e. g. the support, end plate junctions or collector rim (related to the model-induced free-jet deflection) cannot be corrected for.

- An additional slatless configuration with uniform $\left(25^{\circ}\right)$ flap setting was selected as 'low-noise reference' for the slat noise reduction study.

The current report restricts on the presentation of microphone array data with the emphasis on a technology evaluation at wing level. For exemplary farfield noise and background noise data the interested reader might refer to Ref. 22.

According to figure 5 three beamforming scan areas were defined. The largest scan area 1 includes the entire wind-tunnel model and the side plates. This area is meant to (i) assess all kinds of noise sources of the F15LS setup, and (ii) to provide a conservative evaluation of the noise reduction potential of slat setting variations that were applied over the full span of the high-lift system. In case of the adaptive slat only the midboard slat segment of $3 \mathrm{~m}$ span was exchanged, while the remaining slats (outboard upper and lower part) were kept in the reference position. Therefore, evaluation of the noise reduction effect of the two adaptive slat variants was based on the smaller scan grid 2. Finally, the smallest scan area (3) contained only the slat region to evaluate the relative importance of trailing-edge flap and flap track fairing noise contributions. Moreover, this scan area 3 was originally planned for the derivation of 'pure' slat noise validation data (cf. section V.A for the reasons why this had to be postponed to follow-on studies). All grids feature an identical distribution of scan points as a prerequisite for the later comparison of derived sound pressure level spectra. All array data were corrected for wind-tunnel effects (methods identical to those applied for the classical post-processing of farfield microphone data ${ }^{22}$ ). The respective sound pressure levels were referenced towards a constant wing span and constant propagation distance based on spherical sound attenuation. Additional to standard beamforming the CLEAN-SC algorithm by Sijtsma ${ }^{27}$ was applied to the array data to remove excess noise contributions from the spectra. It has to be noted that the current database was collected during the FTEG ${ }^{22}$ /OPENAIR joint DNW-LLF test campaign, which was the first test entry of the newly built F15LS high-lift model. Due to lacking experience with this large-scale model at this point the used experimental setup contains some weaknesses affecting the data quality. Problems that have been solved in subsequent tests with this model were present leakages at the side plate junctions, leading to spurious noise contributions and hence, decreasing the signal-to-noise ratio. Moreover, contrary to the standard slat tracks installed during the OPENAIR tests, alternative streamlined tracks (for fixed reference setting only) mounted at the suction side to reduce slat track noise during validation experiments were in parallel tested in the framework of FTEG.

In figure 5 (right) it is documented that the applied post-processing procedure for array spectra leads to systematic differences in the lower frequency range, when the two different scan areas are used. Overall, 
source maps at frequencies $f<0.8 \mathrm{kHz}$ suffer from a low spatial resolution. Therefore, it is not per se clear which parts of the low-frequency range correspond to slat noise or excess noise contributions, respectively. A tentative analysis of the spectral scaling behavior of corresponding narrowband spectra is provided in section V.A, indicating that spectra derived based on scan grid 1 are contaminated by extraneous noise that could not be fully removed by the CLEAN-SC algorithm. The unequivocal separation of 'pure' slat noise from extraneous noise sources requires further clarification, in particular when using the collected database for high-quality validation purposes beyond the scope of the technical assessment documented in this report. Until then, the major focus has to be set to frequencies in the roll-off region beyond the peak. The slightly raised levels at higher frequencies for scan areas 2 and 3 can be simply explained by a larger number of slat tracks per unit span (leading to an overestimation of these components when normalizing levels on a constant reference span). From the comparison of results derived based on the scan areas 2 and 3 it can be concluded that the trailing-edge flap region does not significantly contribute to the overall wing noise.
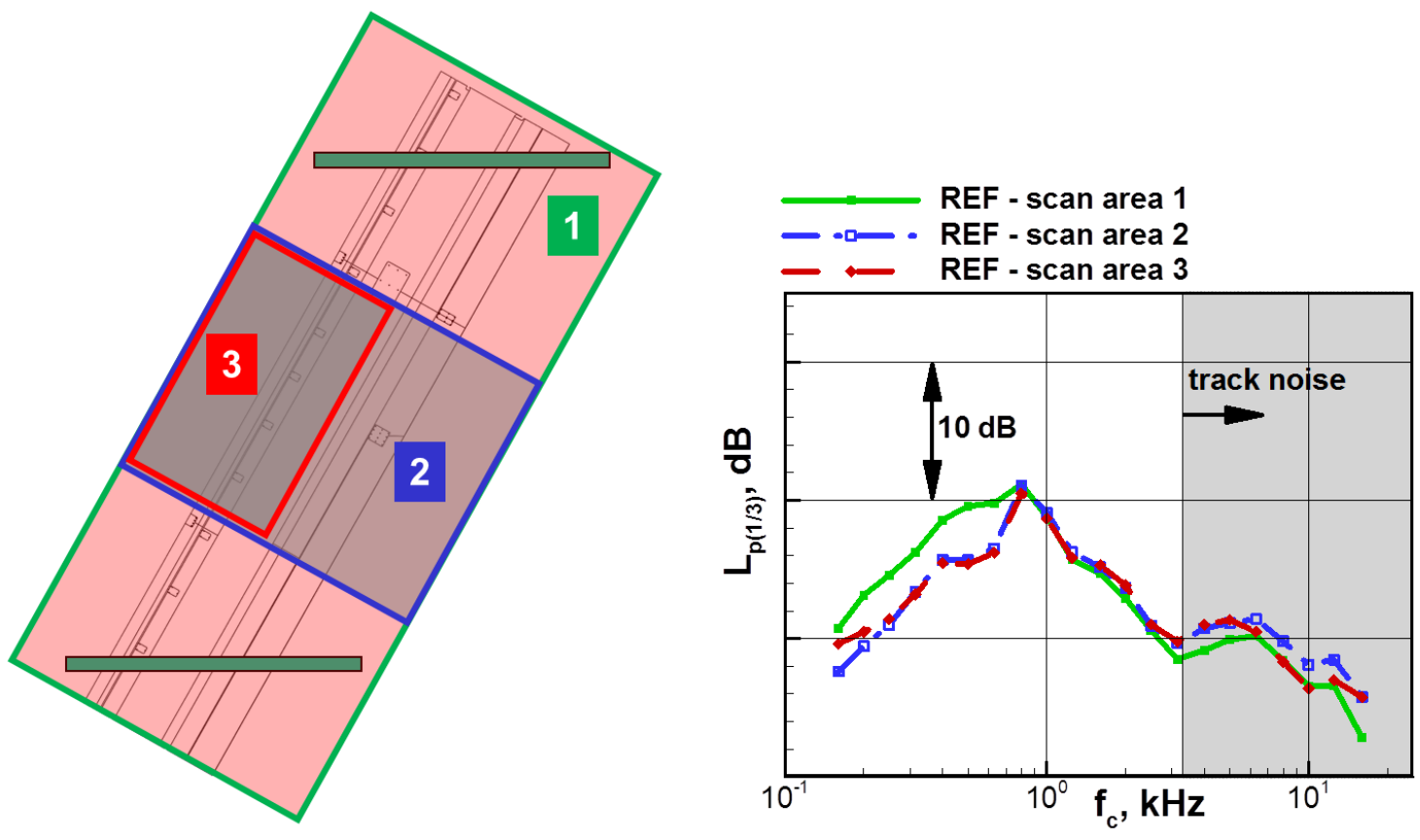

Figure 5. Left: definition of beamforming scan areas. Right: differences observed in the post-processed results, exemplarily shown for reference configuration, $\alpha=5^{\circ}, u_{\infty}=55 \mathrm{~m} / \mathrm{s}, \theta=90^{\circ}$.

\section{Numerical setup}

A hybrid CFD/CAA approach with stochastic source modeling has been applied to provide in addition slat noise simulations for the F15LS high-lift system. The CFD step relies on a solution to the Reynolds Averaged Navier-Stokes (RANS) equations with the DLR code TAU. In the CAA simulation step Acoustic Perturbation Equations (APE) ${ }^{16}$ are solved. For CAA, two different DLR inhouse codes are applied, viz (i) the structured multi-block (SMB) CAA code PIANO ${ }^{7}$ and (ii) the unstructured mesh based CAA code DISCO. ${ }^{1,2}$ Stochastic sound sources are generated with two alternative numerical discretizations of the same stochastic method, i. e. the Random Particle-Mesh (RPM) method ${ }^{15,11}$ and the Fast Random Particle-Mesh (FRPM) method. ${ }^{10,14}$ Sample results from CAA simulations of a 2D section of the F15LS airfoil are subsequently presented, which in total rely on three different combinations of CAA codes with stochastic sources, namely, (i) PIANO with RPM, (ii) PIANO with FRPM, and (iii) DISCO with FRPM. By this procedure it becomes feasible to demonstrate independently from specific questions of numerical discretization an overall idea of the general prediction quality of the RANS/stochastics-based high-lift noise methodology utilized here. Furthermore, this procedure helps to establish an understanding regarding the uncertainties related to different numerical realizations and modeling approaches. Some details to the CAA codes and the stochastic procedure are discussed in the next paragraphs. 

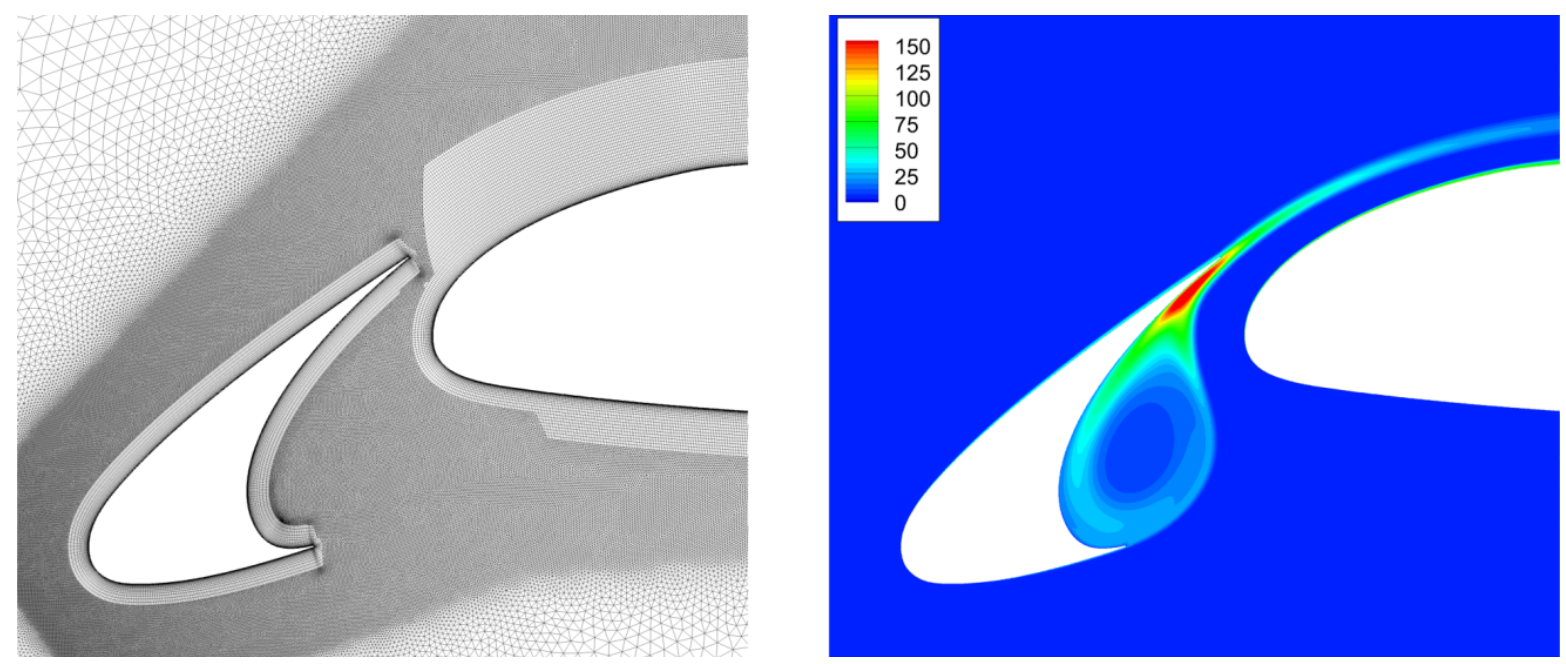

Figure 6. Left: CFD mesh. Right: turbulence kinetic energy (TKE) distribution.

\section{IV.A. CFD simulation}

RANS simulations for all CAA approaches have been computed with DLRs finite volume solver TAU applying a two equation $k-\omega$ SST turbulence model, which provides, besides the mean flow around the slat geometry, also the distributions of turbulence kinetic energy $k$ and specific dissipation rate $\omega$, refer to figure 6 . The simulations are carried out for the F15LS airfoil at the three test velocities and angles of attack. Note, the simulations do not take into account the actual wind-tunnel flow conditions but consider free flight conditions only. All simulations neglect side plates and wind-tunnel parts and the airfoil shows no spanwise change of flow conditions, hence the simulations are pure 2.5D.

Hybrid unstructured CFD meshes were generated with CENTAUR and consist of about 1.1 Mio. points and one cell in spanwise direction. Local refinements around the airfoil and in the slat cove area provide high quality CFD solutions, figure 7(a). Periodic boundary conditions are applied at the spanwise boundaries, thus realizing an infinite wing. The CFD meshes for the PIANO/RPM simulations reported in this paper were generated by means of the SOLAR mesh generator that produces mainly quadrilateral dominant CFD meshes. The SOLAR mesh comprises $670 \mathrm{k}$ mesh points.

\section{IV.B. Stochastic sound sources}

A fluctuating vector force (Lamb vector) is used as a vortex source term in the CAA equations. ${ }^{13}$ A vorticity based source model denoted by 'Source B' is employed in this work, wherein the Lamb vector specifically is modeled from the curl of fluctuating vorticity with the RANS mean-flow velocity,

$$
f=-\Omega^{\prime} \times u_{0} .
$$

Here, $\boldsymbol{u}_{0}$ is the RANS mean velocity and $\boldsymbol{\Omega}^{\prime}$ denotes the fluctuating vorticity, which is generated with FRPM. ${ }^{10,11,14}$ The FRPM method rests on the following two main building blocks:

1. Generation of fluctuating vorticity by superposition of model vortices of random strength and spatial shape (blob) function derived from Gaussian distribution; the vortices advect in a prescribed nonuniform mean-flow field.

2. Efficient numerical realization of the superposition of vortices via random particles and efficient recursive 1-D Gaussian filters applied on an auxiliary FRPM mesh (termed as 'source patch'). 


\section{IV.B.1. Generation of fluctuating vorticity}

In the basic approach ${ }^{\mathrm{a}}$ non-solenoidal and isotropic fluctuating 3D vorticity is realized by the superposition of $P$ random vortex particles,

$$
\Omega_{i}^{\prime}(\boldsymbol{x})=\sum_{p=1}^{P} G\left(\boldsymbol{x}-\boldsymbol{x}_{p}, l_{\Delta}\right) \frac{\hat{A}\left(\boldsymbol{x}_{p}\right)}{\rho_{0}\left(\boldsymbol{x}_{p}\right)} r_{p k}^{n} .
$$

Here, $\Omega_{i}^{\prime}$ is the $i$-th component of a 3D fluctuating synthesized vorticity vector at discrete time level $n$ (dependence on $n$ not explicitly indicated). The summation with index $p$ runs over all random particles. The random particles are represented by their positions $\boldsymbol{x}_{p}$ and carry 3 Gaussian random variables $r_{p k}^{n}$ per particle. Furthermore, $p$ indicates the particle number, and $k$ indicates the random variables, which are Gaussians with vanishing mean, $\left\langle r_{p k}^{n}\right\rangle=0$, and mutually uncorrelated between different particle $(p \neq q)$ and different variables $(k \neq l)$, having a variance defined by $\left\langle r_{p k}^{n} r_{q l}^{n}(t)\right\rangle=\delta_{p q} \delta_{k l} \delta m_{p}$, where $\delta m_{p}=\rho_{0} / \rho_{p}$ indicates a fluid mass fraction related to each particle, which is defined by the mean-flow density $\rho_{0}$ and the particle density $\rho_{p}$ at a specific particle position. The particles passively advect in the local RANS meanflow, i. e. $\dot{\boldsymbol{x}}_{p}=\boldsymbol{u}_{0}$. The particle seeding and distribution is realized so that a particle density $\rho_{p} \propto \rho_{0}$ is obtained and maintained throughout each simulation (i. e. $\delta m_{p}=$ const.). For frozen turbulence $r_{p k}^{n+1}=r_{p k}^{n}$ and the entire source term from Eq. (2) is differentiable in space and time and thus, poses no problem when combined with standard CAA discretization techniques ${ }^{\mathrm{b}}$.

The shape function $G\left(\boldsymbol{x}-\boldsymbol{x}^{\prime}, l_{\Delta}\right)$ defines the realized model vortex associated to each particle. In general, for FRPM a Gaussian distribution is chosen. For 2D CAA simulations, synthetic turbulence is generated with this procedure for just one specific $2 \mathrm{D}$ plane. ${ }^{12}$

\section{IV.B.2. Efficient realization, RPM vs. FRPM}

For the numerical evaluation of Eq. (2) a direct simple computation of the shape function in the surroundings of each particle locations $\boldsymbol{x}_{p}$ is avoided due to its numerical inefficiency. Rather, the random variates of each particle are projected from the particle to an auxiliary background mesh ('source patch') that defines the resolved source area. The Gaussian is evaluated on the source patch via successive application of 1-D Gaussian filtering in each coordinate direction. ${ }^{14}$ Efficient Gaussian filtering is realized with optimized signal processing algorithms, e. g. those proposed by Young \& van Vliet ${ }^{29}$ (constant length scale) or that of Purser ${ }^{23,24,26}$ (variable length scale). Application of the Young \& van Vliet recursive Gaussian filter to the previous example yields a speed up reaching three orders of magnitude saving in computational time compared to simple evaluation of the sum. The FRPM simulations are conducted applying a constant length scale $l_{\Delta}=2.6 \cdot 10^{-3} l_{c}$. Refer to figure $7(\mathrm{c})$ for the location of the FRPM source patch.

RPM is a precursor implementation of the procedure FRPM method, which is only applicable in 2D. For RPM a mean-flow streamline based background mesh is used to realize a spatially and temporally resolved turbulence field, figure 8 (left). To assure maximal comparability this background mesh was built always in an identical manner. The distance of the first streamline to the slat trailing edge was $5 \cdot 10^{-4} l_{c}$ and the distance of the last streamline to the main element in the slat gap was $2 \cdot 10^{-3} l_{c}$. The remaining gap was then discretized by 30 streamlines. The first streamline started at the slat hook and the length of the first streamline was $0.3 l_{c}$ discretised with 500 points. The source area is visualized in figure $8(\mathrm{a})$. Advecting particles are realized by seeding particles with a constant clock rate of $\Delta t=3 \cdot 10^{-3} l_{c} / c_{0}$, where $c_{0}=341 \mathrm{~m} / \mathrm{s}$ is the speed of sound, which gives an average of approximately 500 particles per streamline. The Gaussian filter step is already split into streamwise and lateral directions to avoid a simple summation. A constant length scale $l_{\Delta}=4 \cdot 10^{-3} l_{c}$ is applied.

\section{IV.C. Simulations with DLR code DISCO}

The Discontinuous Galerkin CAA-code DISCO works on unstructured grids consisting of triangular elements and solves Acoustic Perturbation Equations. ${ }^{16}$ DISCO has $4^{\text {th }}$-order spatial accuracy utilizing Lagrangian polynomials as shape functions with 10 degrees of freedom (DOFs) per triangular element. Mean-flow

\footnotetext{
${ }^{\mathrm{a}}$ An extension to anisotropic 3D and divergence-free (solenoidal) is presented by Reiche et al. ${ }^{25}$

${ }^{\mathrm{b}}$ Decaying turbulence with specific decay time can be realized by defining the random variable by means of an OrnsteinUhlenbeck process (Langevin equation) with according time scale. ${ }^{14}$
} 


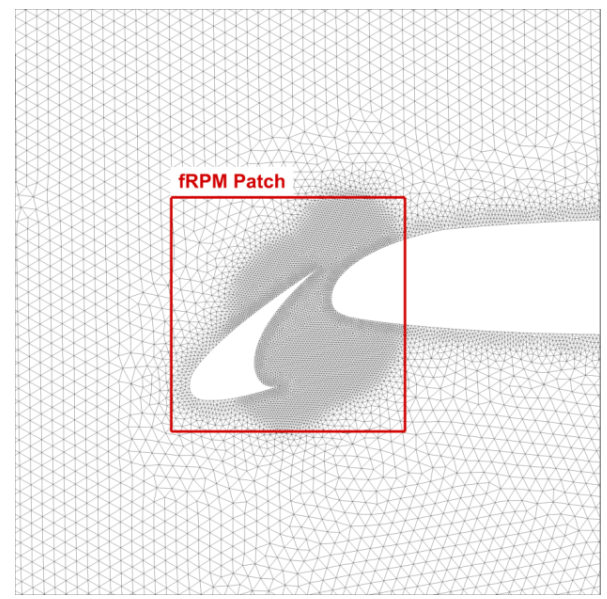

(a) DISCO CAA mesh with FRPM patch.

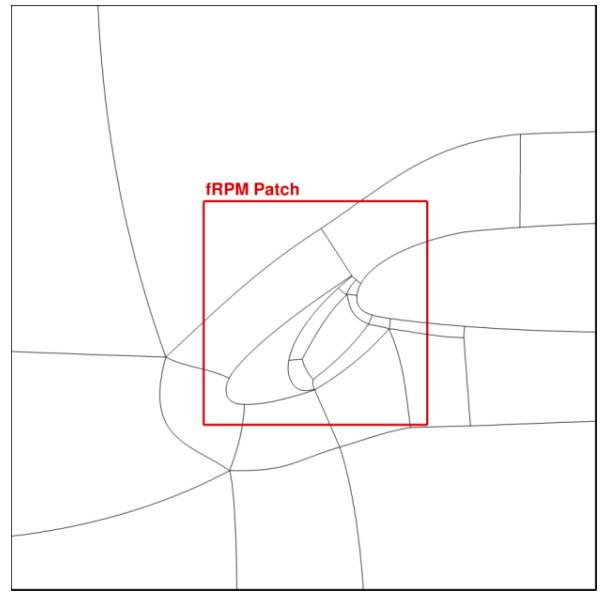

(c) PIANO CAA mesh with FRPM patch.

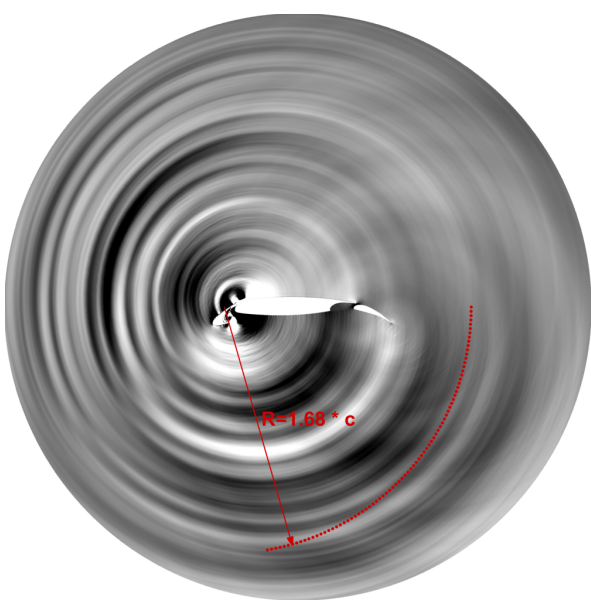

(b) Unsteady pressure field with virtual microphone positions.

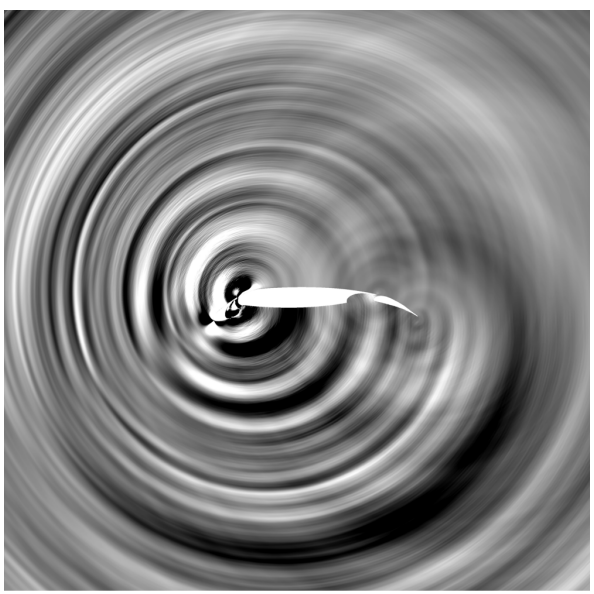

(d) Unsteady pressure field.

Figure 7. Comparison of DISCO and PIANO meshes and solutions.

gradients are properly taken into account. A $4^{\text {th }}$-order accurate non-uniform time step scheme (NUTS) ${ }^{20}$ is applied for temporal integration, which typically yields a speed-up of approximately factor 10 for the high-lift geometry compared to standard $4^{\text {th }}$-order explicit time integration. DISCO is coupled to the FRPM module to model the turbulent source term.

The circular 2D CAA meshes are as well generated using the mesh generator CENTAUR, consist of triangles and are designed to resolve a maximum frequency of $10 \mathrm{kHz}$. For the $4^{\text {th }}$-order elements with 10 degrees of freedom (DOF) 2-3 elements per wavelength are needed for a good resolution, resulting number of $\sim 1.5$ Mio. points. A number of 81 virtual microphones are positioned on a polar arc in the lower rear of the slat with a radius of 1.68 chord lengths around the nose point of the retracted slat.

Figure 7(a) shows details of the DISCO/FRPM mesh at the slat and figure 7(b) presents a snapshot of the sound field.

\section{IV.D. Simulations with DLR code PIANO}

The PIANO code works on structured multi-block meshes applying the $4^{\text {th }}$-order accurate Dispersion Relation Preserving (DRP) scheme proposed by Tam \& $\mathrm{Webb}^{28}$ and a $4^{\text {th }}$-order Runge-Kutta method for explicit time integration. More details of the method as applied to the slat problem can be found in Ref. 13.

For PIANO/FRPM simulations a 2D block-structured CAA mesh is generated using the mesh generator Pointwise. The mesh consists of 56 structured bocks and is designed to resolve frequencies up to $15 \mathrm{kHz}$ with 7 points per wavelength resulting in $\sim 2.4$ Mio. points. 

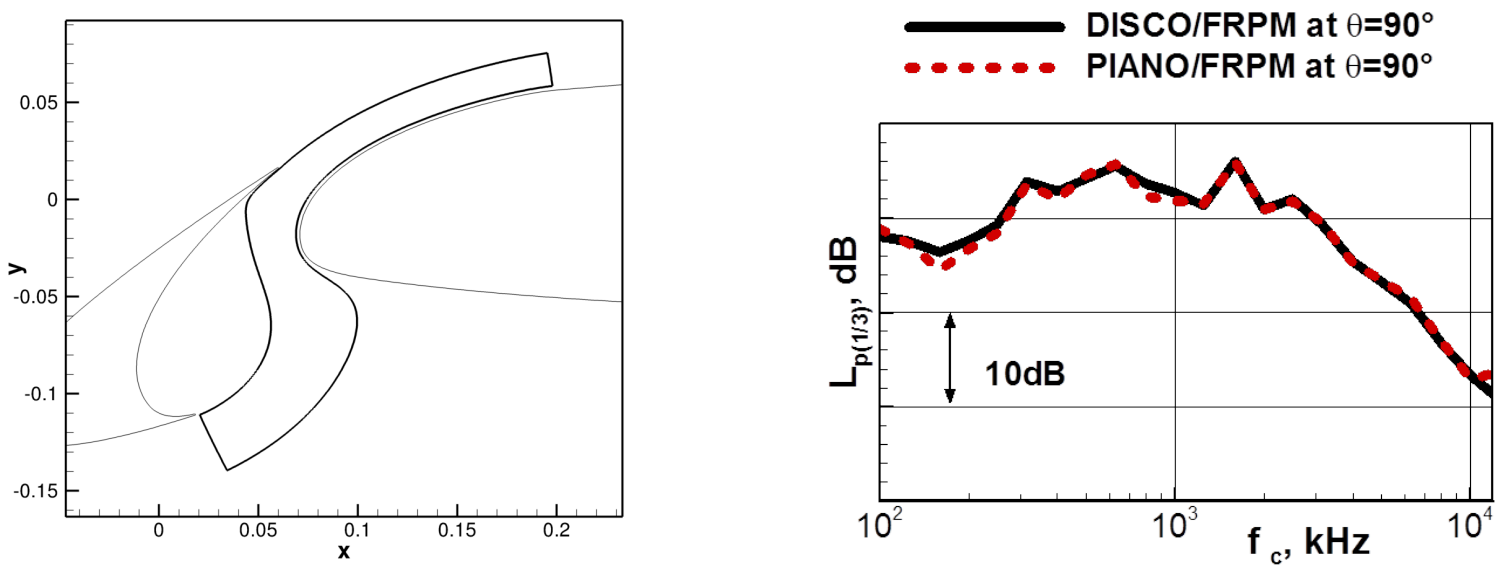

Figure 8. Left: RPM source patch location at the slat. Right: simulated 1/3-octave band spectra. Comparisons of PIANO/FRPM and DISCO/FRPM results.

For the PIANO/RPM simulations a CAA mesh with $633 \mathrm{k}$ points and 54 blocks is used, also designed for $15 \mathrm{kHz}$ highest resolved frequency. Figure 7(c) shows details of the PIANO/FRPM mesh at the slat and figure $7(\mathrm{~d})$ presents a snapshot of the sound field. Figure 8 (right) shows a comparison of one-third octave spectra predicted with PIANO/FRPM and DISCO/FRPM at polar angle $90^{\circ}$ above the slat. No significant difference is visible between the predictions obtained with both CAA codes. Due to the close agreement between both approaches, this paper mainly focuses on a presentation of results as obtained with the DISCO/FRPM compound.

\section{Results}

\section{V.A. Preliminary remarks on the reference slat noise data}

Figures 9 and 10 provide narrowband spectra as measured with the microphone array for the three test velocities and angles of attack for an 'overflight' observation angle of $\theta=90^{\circ}$. Post-processing was based on the array scan areas 1 (full wing) and 3 (slat only), respectively. A normalization according to a $4.5^{\text {th }}$ power velocity-law $^{8,9}$ was applied, i. e.

$$
\begin{aligned}
L_{\text {pnorm }} & =L_{p}-45 \log \left(M_{\infty}\right) \\
S t & =f l_{s} / u_{\infty} .
\end{aligned}
$$

Herein, normalization is based on the freestream Mach number $M_{\infty}$ and the slat chord length $l_{s}$ as relevant length scale.

For both scan areas, strong tonal components are observed in the reference noise spectra. These components increase with decreasing angle-of-attack and appear in the region where typically broadband slat noise spectral peaks are located (at $S t \approx 2$ according to Refs. 8,9). The observation of tones was not according to expectation, given the high test $R e$ in the current experiment along with the previously published Re-dependence of tones in slat noise experiments. ${ }^{22}$ To precise, the mentioned velocity study in Ref. 22 was conducted with the same model but based on a different slat setting configuration. This confirms the tentative conclusion in Ref. 22 that fundamentally different tone generation mechanisms might be active. Unfortunately, a final clarification of the underlying relationships was beyond the scope of the OPENAIR project and will have to be subject to further analysis of the available F15LS database. A future detailed investigation of the appearance of tones in dependency of geometry and flow parameters should additionally include newer F15LS test data with improved signal-to-noise ratio (see section III.B).

The noise assessment in OPENAIR focused at an evaluation of noise differences at wing level, taking into account also measured farfield noise directivities (not shown herein). Data from a first straightforward assessment, i. e. based on test data derived with scan area 1 (and scan area 2 for the adaptive slat configurations) are documented in the remainder of this paper. When selecting area 1, tonal components are partly 

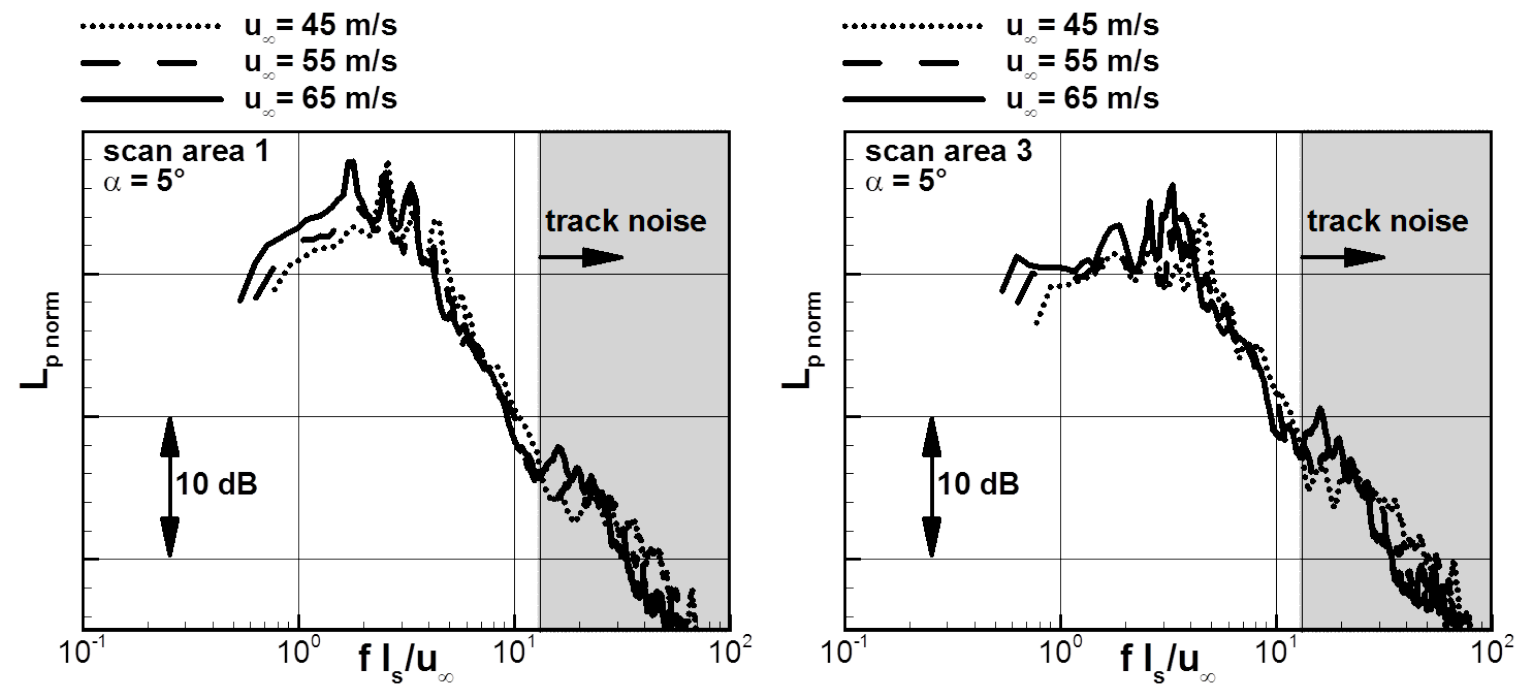

Figure 9. Velocity scaling behavior of narrowband spectra based on variant array scan areas, $\alpha=5^{\circ}, \theta=90^{\circ}$.

buried and observed trends consistently correspond to the measured farfield noise characteristics with noise differences predominantly characterized by broadband slat noise contributions. Remind that the results in the roll-off region beyond the peak are neither affected by tones nor by the choice of the integration area. This respective frequency region will contribute most to the higher A-weighted spectral range when transposed to full scale. However, as shown in figure 9, the velocity scaling behavior of the broadband spectral contents looks less perfect for scan area 1 at lower frequencies which is likely due to the presence of extraneous noise sources (e.g. caused by the side plates) that cannot be fully removed from the spectra by application of CLEAN-SC. This subject has been brought up already in Ref. 22. Particularly for the planned provision of high-quality validation data for CAA-based slat noise predictions the data post-processing will require further refinement. The latter, however, should be based on a preceding analysis on the relevance of the observed tone phenomena and should then, after clarification, limit on the smaller scan area 3 to ensure that any other noise contributors than the slat are removed from the analysis.

\section{V.B. Noise reduction potential of low-noise slat technologies}

Major test results are presented in the following key figures 11 and 12, wherein measured noise reductions are shown in terms of $1 / 3$-octave band spectra, evaluated at wing level (array data based on scan areas defined above). These selected viewgraphs are restricted to representative spectra for $\theta=90^{\circ}, \alpha=5^{\circ}$, and $u_{\infty}=$ $55 \mathrm{~m} / \mathrm{s}$. Note that the same conclusions can be drawn for the other test conditions, being supported by equivalent trends observed in the farfield noise data. To avoid doubling in the presentation of measurement data a more detailed discussion of individual trends observed for systematical parametrical changes (like gap reduction at constant overlap or overlap variations at constant gap, respectively) will be provided in section V.C along with the numerical data presentation. Overall, the DNW-LLF test results for the low-noise slat technologies have proven a promising noise reduction potential with acceptable aerodynamic impact, as further specified below.

\section{V.B.1. Adaptive slat}

As highlighted in figure 11, the adaptive slat (closed gap) provides a broadband noise reduction of order $5 \mathrm{~dB}$ at wing level, equivalent to a full elimination of the slat noise source. Here, the slatless 'low-noise reference' provides a rough estimate of the lower bound, even though local flow conditions are not identically comparable. Data at $f<0.8 \mathrm{kHz}$ for the adaptive slat configuration are displayed as dashed lines due to the uncertainties discussed in sections III.B and V.A. For a conservative estimate of the noise reduction at these lower frequencies refer to the equivalent reference noise spectrum derived based on array scan area 2 (see figure 5). The concept of the adaptive slat showed a lift penalty at the main element for the three test 

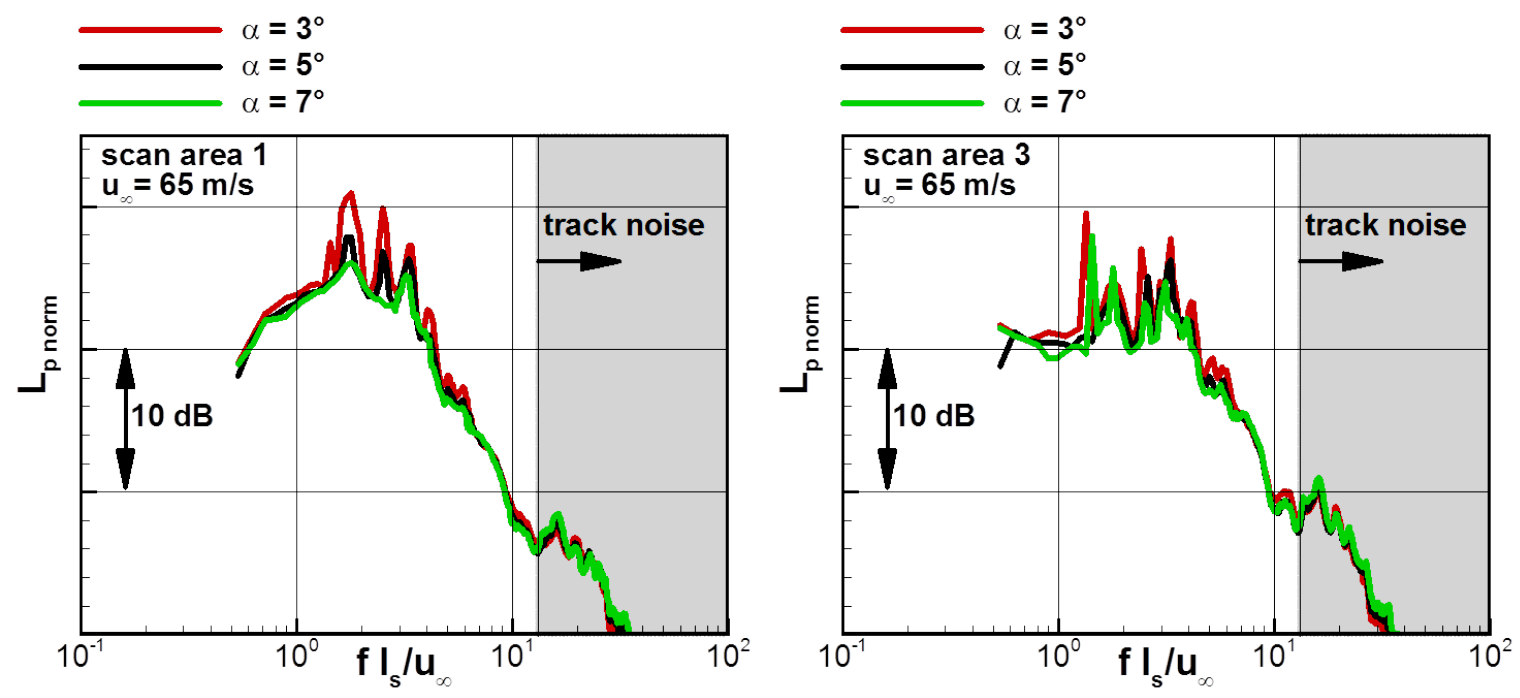

Figure 10. Narrowband spectra for the three test angles-of attack, $u_{\infty}=65 \mathrm{~m} / \mathrm{s}, \theta=90^{\circ}$.

angles-of-attack, as documented in terms of measured distributions of the normal force coefficients $c_{n}$. Note, however, that an incomplete picture is drawn due to the lacking instrumentation of the morphed slat part. Corresponding CFD simulations (not shown herein) revealed a lift increase at the slat, associated by a lift decrease at the main element, resulting in an overall preserved total lift within the linear region of the polar. A loss in maximum lift does not exist by definition of the concept (emergency release system that resets the original reference shape and gap/overlap setting).

\section{V.B.2. Slat setting variations}

Figure 12 documents that modified slat settings (or an adaptive slat with intermediate gap width, cf. figure 11) are suited to reduce slat noise by about $2-3 \mathrm{~dB}$ at wing level while producing negligible aerodynamic impact at the operative test angles of attack. Specifically, a lift increase at the slat that counterbalances the lift decrease at the main element was measured. CFD results correspond to this findings. A good reproduction of the (small-scale) TIMPAN results was obtained also at the large-scale swept configuration. Namely, the relatively broad optimum for low-noise slat settings (cf. figure 1) was confirmed, i. e. slat settings SO2, $\mathrm{SO} 3$, and $\mathrm{SO} 4$ provide a similar noise reduction potential. Overall, the new OPENAIR setting SO4 provides the best acoustic performance even though observed differences are very small.

\section{V.B.3. Projection of the wind-tunnel test results to flight conditions}

Estimates of corresponding effects on the aircraft Effective Perceived Noise Level (EPNL) under approach conditions were derived based on the well-established Airbus Technology Evaluator ${ }^{4}$ process. Assuming a typical short-to-medium range aircraft configuration an overall 0.5 -EPNdB noise reduction is considered achievable by slat setting optimization. When a preliminary assessment of integration affects is accounted for, this result would be reached by both the best slat setting SO4 and the adaptive slat (noise assessment based on closed gap variant). This result complies with the defined OPENAIR target to achieve an overall $3.2-\mathrm{dB}$ noise reduction of wing noise at approach. The overall 0.5-EPNdB noise reduction result rests on the assumption that all other noise sources at the aircraft than the slat remain untreated and that the gap reduction does not shift the stall onto the outboard wing. Evaluation of the adaptive slat was based on measured noise differences, when using the beamforming scan area 2 for both the adaptive slat and the reference configuration. 

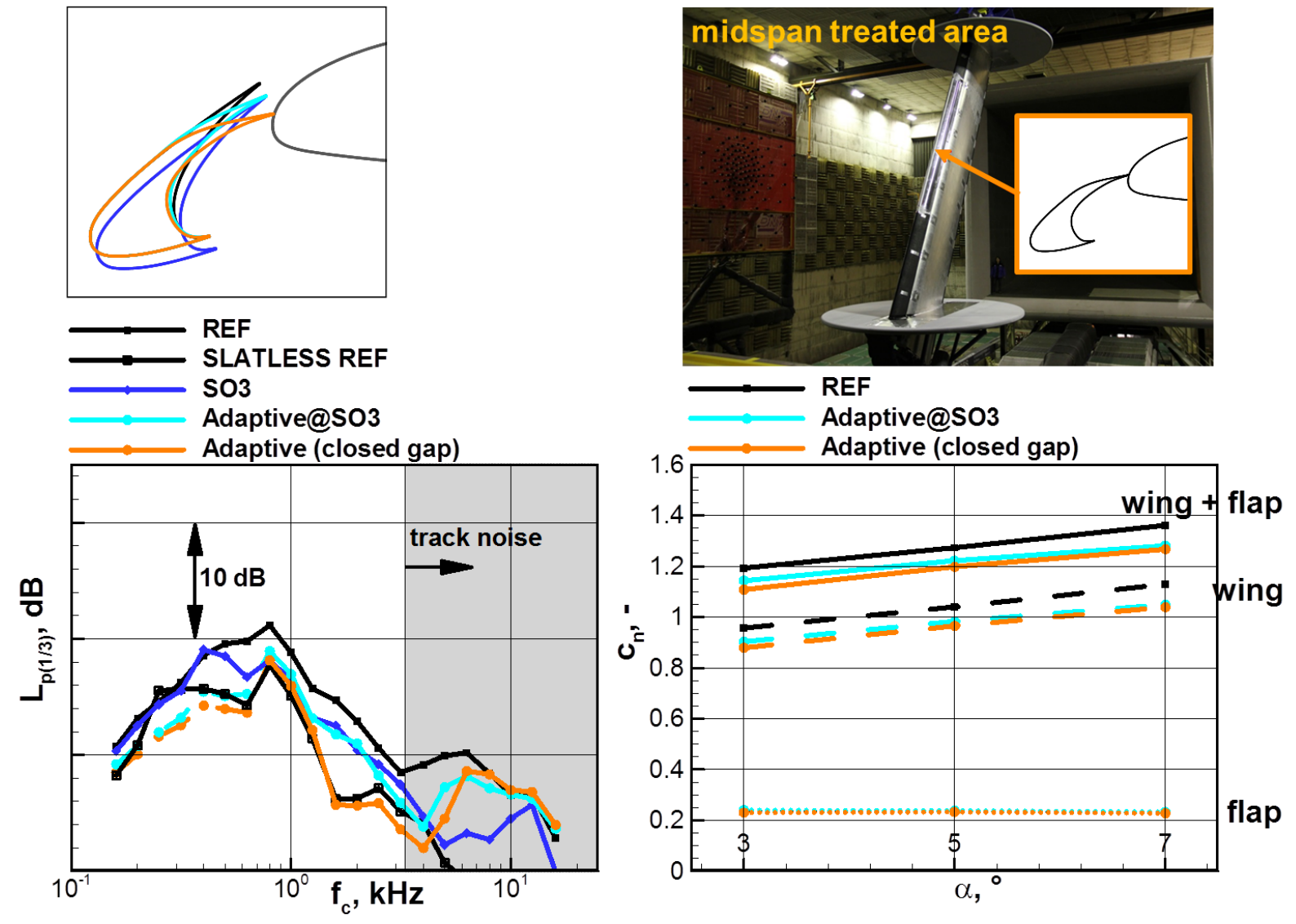

Figure 11. Assessment of the adaptive slat technology. Left: array results at $\theta=90^{\circ}, \alpha=5^{\circ}$, and $u_{\infty}=55 \mathrm{~m} / \mathrm{s}$. Right: $c_{n}$-distribution along the midspan wing section for the three test angles-of-attack, $u_{\infty}=55 \mathrm{~m} / \mathrm{s}$. 

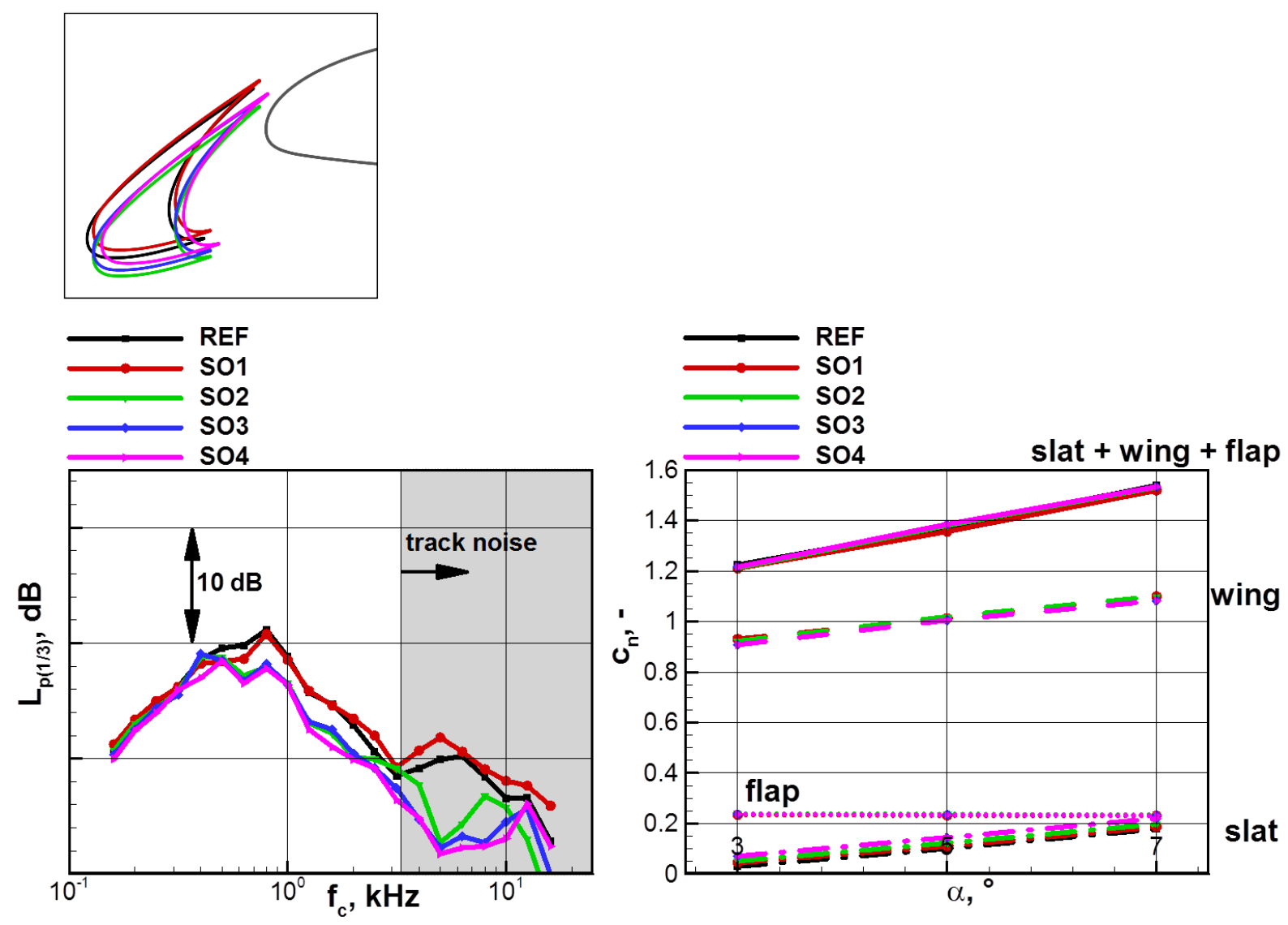

Figure 12. Assessment of the slat settings SO1-SO4. Left: array results at $\theta=90^{\circ}, \alpha=5^{\circ}$, and $u_{\infty}=55 \mathrm{~m} / \mathrm{s}$. Right: $c_{n}$-distribution along the midspan wing section for the three test angles-of-attack, $u_{\infty}=55 \mathrm{~m} / \mathrm{s}$. 


\section{V.C. Comparison of measurement data with numerical predictions}

\section{V.C.1. Spectral characteristics}

Prior to a more detailed overview of predicted spectral shapes and observed trends a direct code-to-code comparison of results is provided in figure 13. Here, 2D DISCO/FRPM and PIANO/FRPM predictions for the OPENAIR settings SO1-SO3 are compared with measurement data in in terms of narrowband spectra.

Equivalent prediction results were obtained for $f<5 \mathrm{kHz}$. Systematic deviations visible at higher frequencies can be traced back to differences in the resolution of the slat trailing-edge region with both CAA meshes. Note that - similar to the shown comparison in figure 8 (right) - the presented results were independently produced by different operators applying individual CFD inputs, which underlines the robustness of the used method. As has been for example documented in Ref. 1 identical CFD and FRPM setups will generally provide identical prediction results.
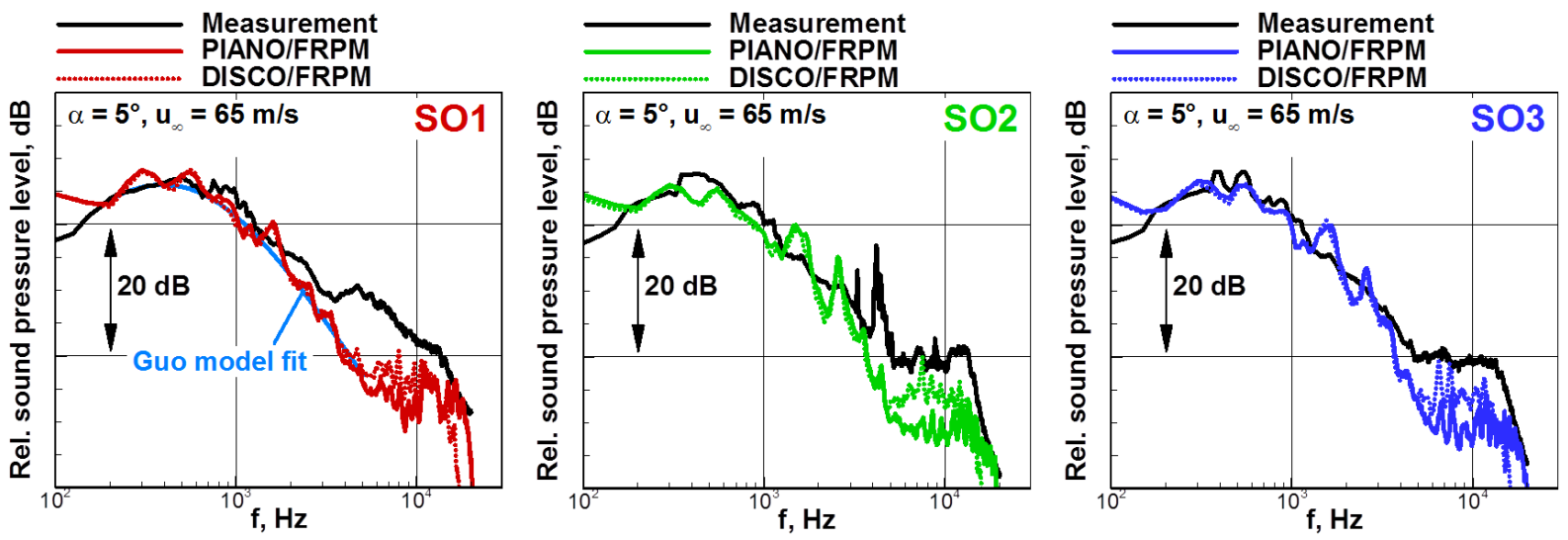

Figure 13. Comparison of DISCO/FRPM and PIANO/FRPM predictions with microphone array data (narrowband spectra). OPENAIR configurations SO1-SO3, $\theta=90^{\circ}$.

Spectral characteristics are well-reproduced for broadband slat noise in a frequency range from approximately $0.2-3 \mathrm{kHz}$, whereas noticeable differences restrict to tonal features in both the measurement and simulation data. Pronounced frequency-fixed $1.5-\mathrm{kHz}$ tonal components are visible in the simulated spectra. These are identically expressed in all results and represent 2D-effects that are not observed in equivalent 3D PIANO simulations. Note that the corresponding wave length of $\lambda=0.226 \mathrm{~m}$ matches the slat chord length of $l_{s} \approx 0.23 \mathrm{~m}$. At frequencies $>3 \mathrm{kHz}$ measured spectra provide a hump/plateau at higher frequencies which is dominated by slat track noise which cannot be clearly extracted from the measurement data. Simulations suggest a steeper roll-off in levels at those frequencies; the spectral slopes being present in the narrow band spectrum in a frequency range above the spectral maximum is proportional to $f^{-2}$ and gets steeper for higher frequencies, until finally reaching a slope proportional to $f^{-5}$. The decay corresponds with that prescribed by the characteristic slat noise spectrum proposed by Guo ${ }^{17,18}$ (exemplarily plotted for SO1 in the figure). In addition to that, simulations provide lower-level plateaus/humps at high frequencies approaching the grid resolution cut-off frequency ( $15 \mathrm{kHz}$ in all of the shown cases). Due to the measurement data uncertainties in this range a final statement concerning their physical and/or numerical origin will have to be left to future studies.

Given the very good matching with the PIANO predictions, albeit with significantly simplified meshing effort, DISCO reveals certainly advantages for industrial use. Artificial sharpening of the slat trailing-edge and cusp (i. e. avoidance of a clustering of grid points) significantly reduces the necessary amount of time steps without significantly lowering the prediction quality. Correspondingly adapted simulations required only 15 hours computation time on a standard workstation without parallelization applied utilizing a non-uniform time step method, refer to section IV.C.

\section{V.C.2. Observed trends}

Comparisons in the following figures are all based on a one-third octave band representation of spectra. Figure 14 provides an overview of spectra for 6 different test configurations that were selected for simulations. Again, measured and predicted spectra are presented in a normalized manner as ${ }^{8,9}$ 

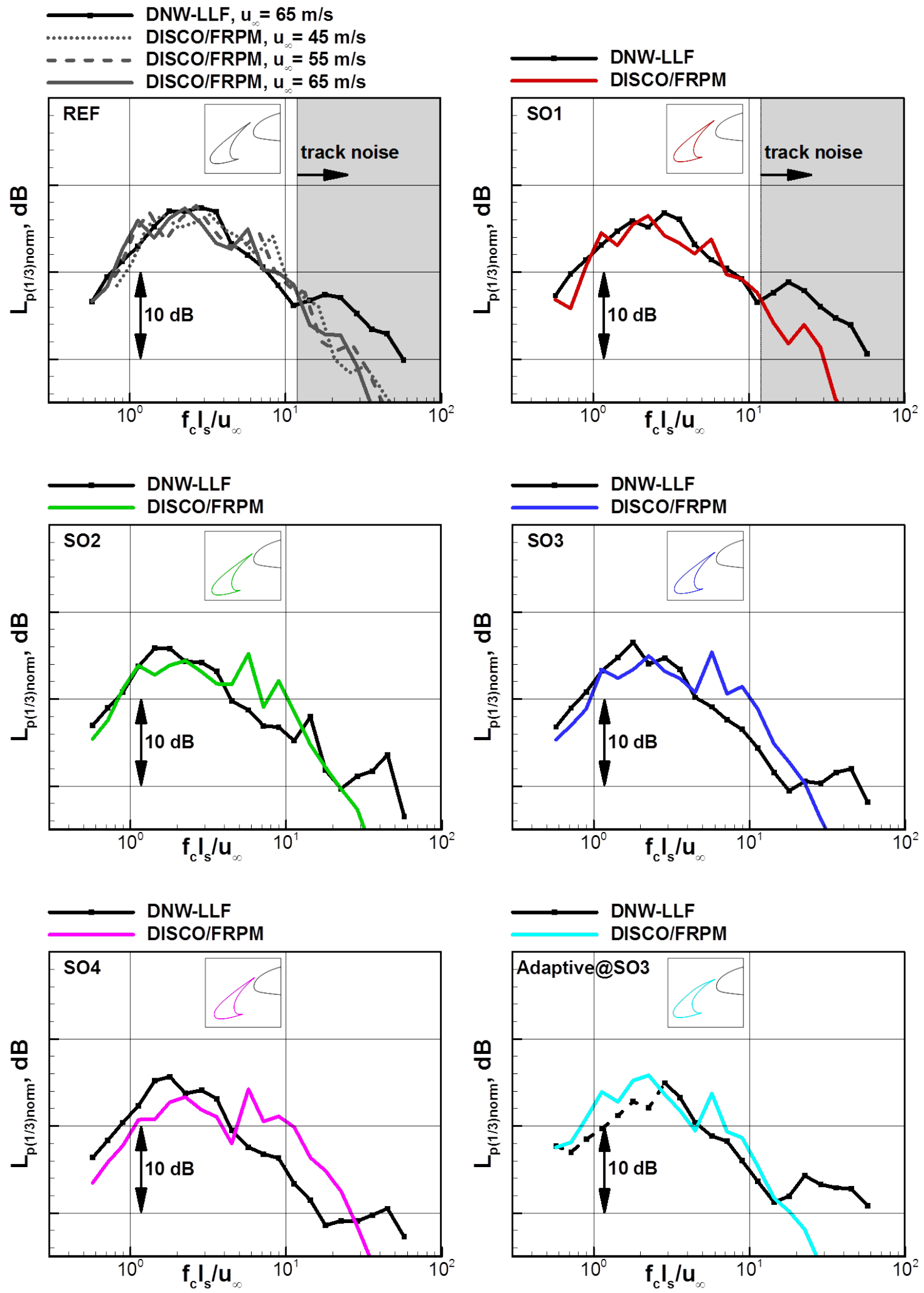

Figure 14. Comparison of predictions with microphone array data for individual test configurations, $\alpha=5^{\circ}, u_{\infty}=$ $65 \mathrm{~m} / \mathrm{s}$ (if not otherwise noted), $\theta=90^{\circ}$. 


$$
\begin{aligned}
L_{p(1 / 3) \text { norm }} & =L_{p(1 / 3)}-45 \log \left(M_{\infty}\right) \\
S t & =f_{c} l_{s} / u_{\infty} .
\end{aligned}
$$

As shown for the reference configuration (top left) the expected velocity scaling behavior of slat noise is perfectly reproduced by the CAA simulation results. For completeness, correspondingly unscaled data are additionally documented in figure 15 (left).

Again, discrepancies between measurement and prediction results are observed in the high-frequency region, where the DNW-LLF test data contain excess noise contributions mainly caused by the slat tracks. These contributions in the measured spectra tend to decrease when the gap width and corresponding effective flow velocities are reduced. Overall, the general spectral shape, featuring a noise maximum at $S t \approx 2$ (in accordance with Refs. 8,9), is fairly well reproduced. However, remind the limitations of the experimental data at frequencies close to the spectral maximum and below. Unfortunately, the mentioned 2D tonal features in the simulated spectra dominate the $1.5-\mathrm{kHz}$ frequency band and also aggravate the evaluation of broadband noise reduction at adjacent frequencies, particularly for the low-noise configurations. Since the DNW-LLF comparison data obtained for different array integration areas provide best comparability at frequencies in the spectral roll-off region above the spectral peak, fully 3D-computations might be preferred later for downselected configurations if more detailed information in this particular frequency range is requested.

Figure 15 (right) shows the effect of angle-of-attack on unscaled slat noise spectra obtained for the reference slat setting. Except for the spectral scatter in the peak region (related to the tonal components documented in figure 10), measurements revealed a negligible impact of the test angle-of-attack on broadband slat noise. This broadband behavior, as shown in the figure, is well-represented by the CAA results.

For the slat setting variations (OPENAIR settings SO1-SO4) and adaptive slat (intermediate gap width) also a generally good prediction of major trends is observable in the following figures 16 to 18 . In these viewgraphs the above datasets from figure 14 are reformatted to illustrate major trends in both the measured and predicted spectra. Figure 16 presents the effect of overlap variations (at constant gap settings), whereas figures 17 and 18 (left) extract the effect of a changed gap width (at constant overlap settings). Moreover, potential 'shape effects' induced by the adaptive slat morphed shape (compared to the conventional slat at same gap/overlap setting) are presented in figure 18 (right).

To summarize from these overviews, on overlap increase has a negligible effect on measured spectra, as documented in figure 16. In contrast, deduced from figures 17 and 18, the gap width is clearly identifiable as the driving parameter to achieve a significant noise reduction. In other words, major results from the TIMPAN project ${ }^{13}$ could be reproduced in OPENAIR, i. e. were found valid also for a swept wing configuration at much higher Re. Particularly, the effect of gap width reduction is clearly visible in the prediction results. Corresponding distributions of the kinetic energy of turbulence $k$ are added in the figure (top left), indicating smaller regions with increased values of $k$ when reducing the gap width. For a better view also a directivity plot, where the tonal artefacts have been omitted in the integration range, is presented. It is worth to recall that the preselection of the most effective setting variant ( $\mathrm{SO} 4$ according to both the measurements and the DISCO/FRPM simulations) in OPENAIR has been made feasible by preceding CAA-assisted design recommendations, as it is briefly discussed in section I.A. According to figure 18 (right) a distinctive adaptive slat 'shape effect' cannot be deduced from neither the measurement nor the simulation results.

Obviously, measured differences to be resolved were relatively small for most of the tested slat setting variants, supporting the expectation of a relatively broad optimum for aeroacoustically optimized slat settings as deduced from the TIMPAN data (cf. figure 1). The latter is considered beneficial in terms of technical realization on the one hand. On the other hand, resolution of relative differences $<2 \mathrm{~dB}$ represents a challenge in both measurements and simulations. CAA results slightly underpredict (SO2, SO3 compared to SO1) or overpredict the measured deltas ( $\mathrm{SO} 4$ compared to $\mathrm{SO} 2$ ). The contained scatter in the predictions might be best illustrated by comparisons with earlier simulation results that were produced within the PIANO-RPM framework $^{3}$ (utilizing also different CFD/RANS inputs). A selection of configuration overviews, presented in the same data format as the above comparisons, is shown in figure 19. Here, it is notable that the predicted differences for gap closure are much more expressed than in the previously discussed results. Both shown older PIANO-RPM and new DISCO-FRPM results provide the bracketing range in which the measured dependencies are observed. As one example reverse trends are documented for the comparison between the settings SO2 and SO4, while measurements indicate only a marginal difference in the spectra for these two configurations. Future work will therefore concentrate on the definition of the most suitable numerical best practice setups to further enhance the realizable resolution in the prediction of noise differences. 

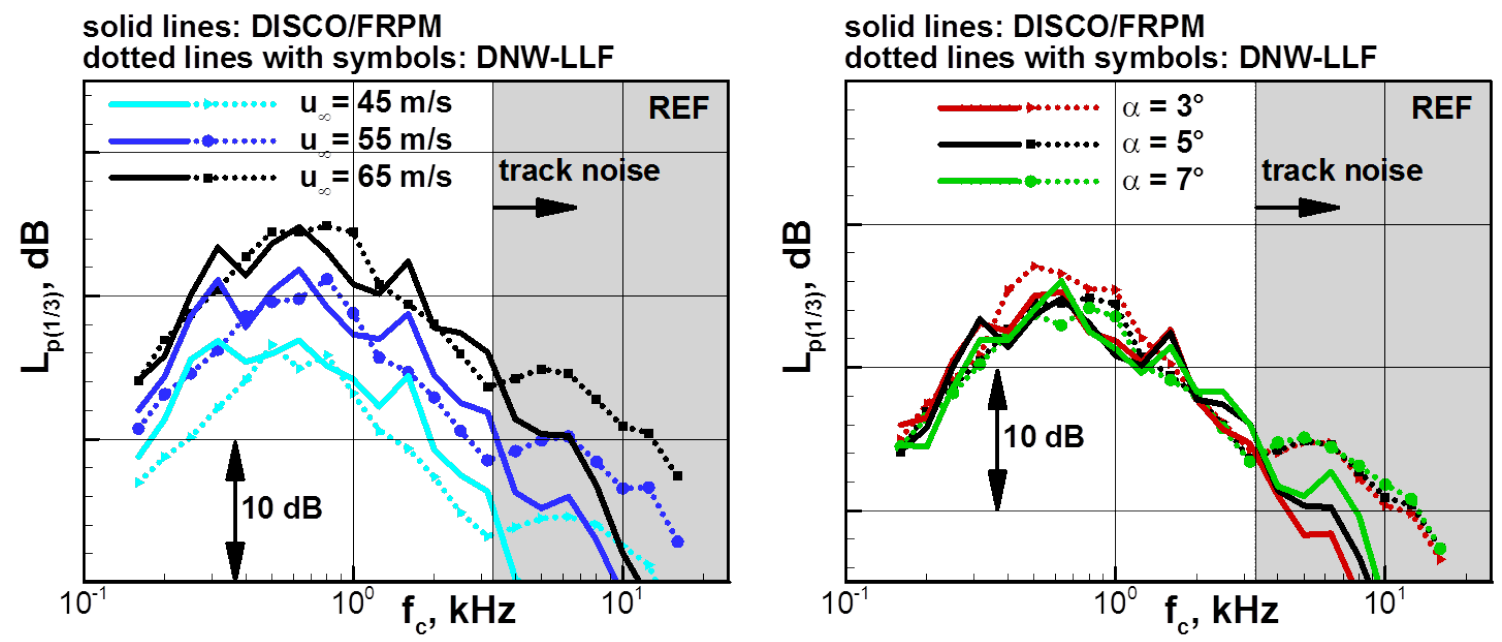

Figure 15. Comparison of predicted and measured trends. Left: effect of flow velocity $u_{\infty}$; reference configuration, $\alpha=5^{\circ}, \theta=90^{\circ}$. Right: effect of angle of attack $\alpha$; reference configuration, $u_{\infty}=65 \mathrm{~m} / \mathrm{s}, \theta=90^{\circ}$.
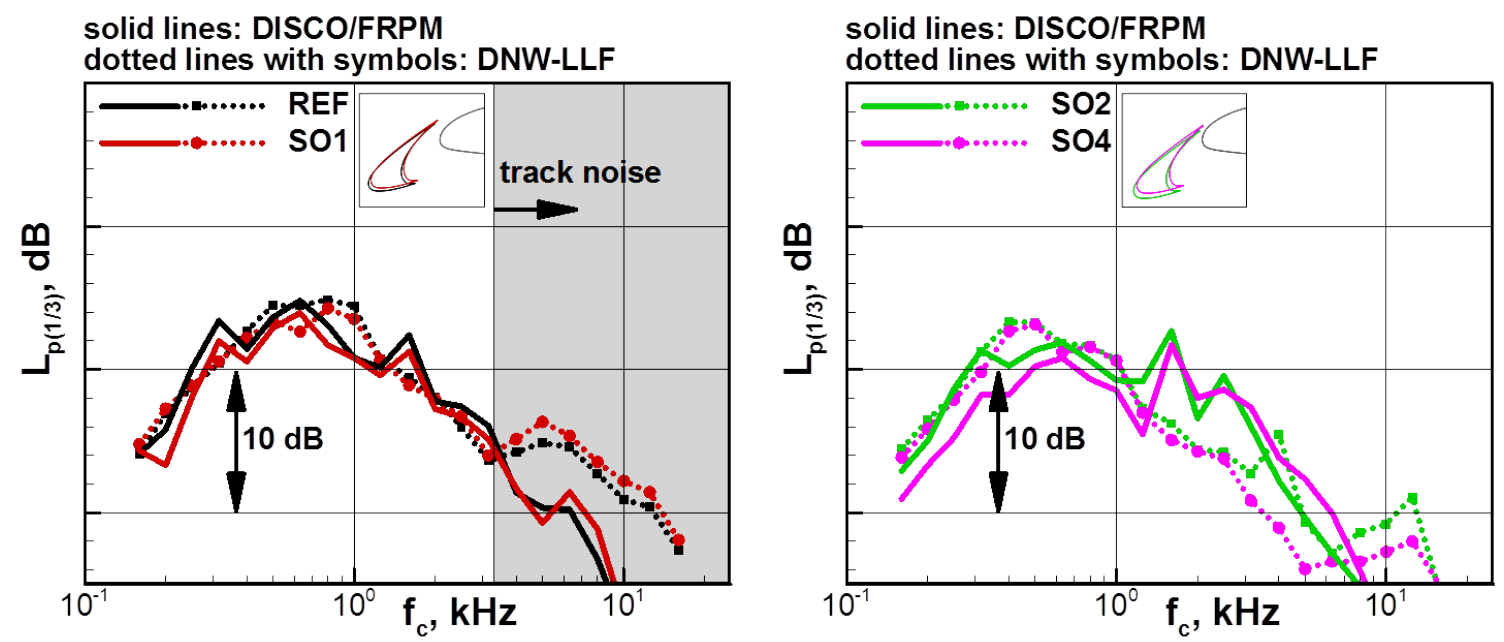

Figure 16. Comparison of predicted and measured trends. Effect of overlap variation; conventional slat with constant gap setting, $\alpha=5^{\circ}, u_{\infty}=65 \mathrm{~m} / \mathrm{s}, \theta=90^{\circ}$. Left: reference configuration vs. SO1. Right: SO2 vs. SO4. 

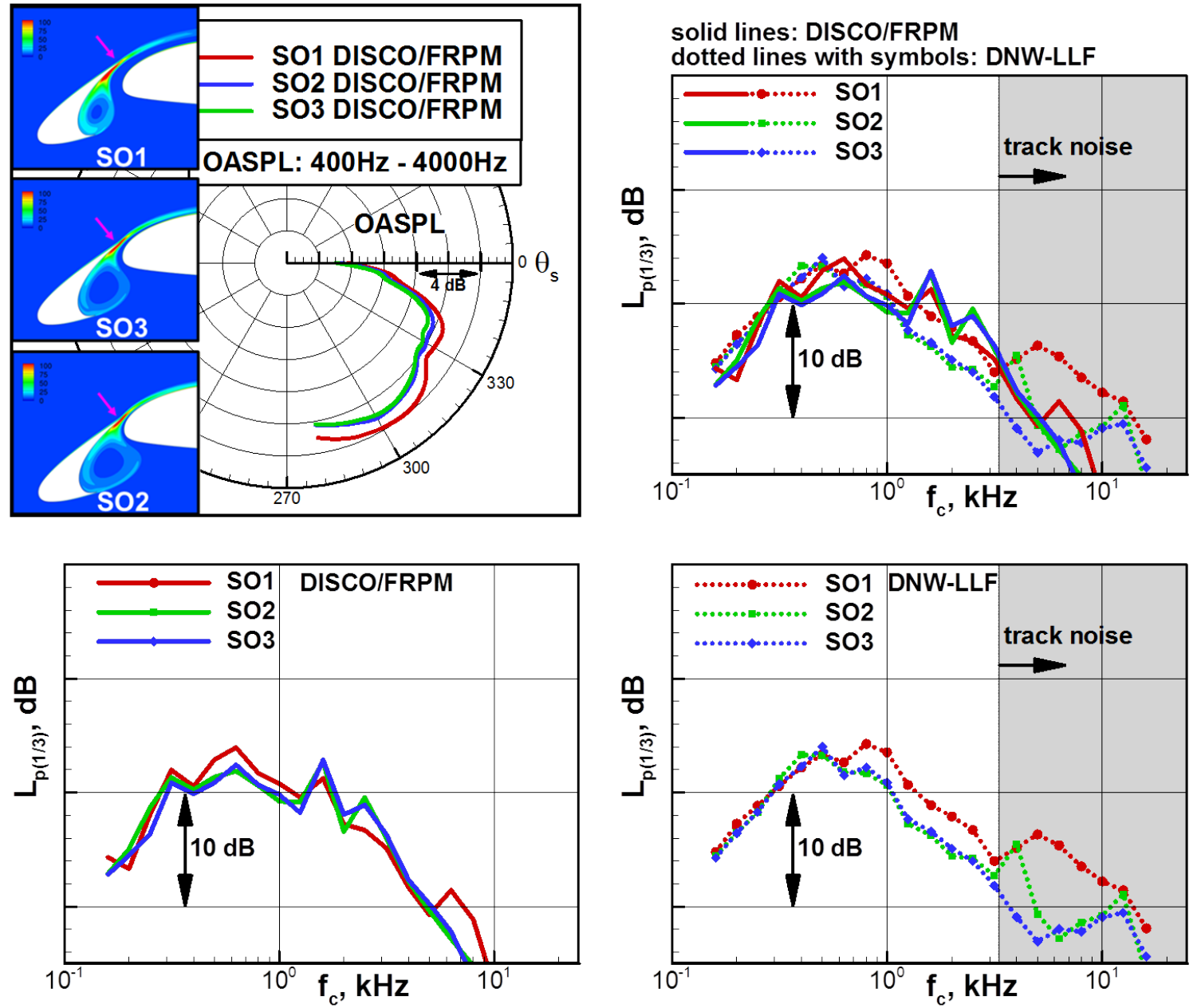

Figure 17. Comparison of predicted and measured trends. Effect of gap variation; conventional slat with constant overlap setting, $\alpha=5^{\circ}, u_{\infty}=65 \mathrm{~m} / \mathrm{s}, \theta=90^{\circ}$. Top left: simulated distributions of kinetic energy of turbulence and overall sound pressure level (OASPL) directivities. Top right: measurements and predictions for OPENAIR settings SO1-SO3. Bottom: same datasets in separate graphs for predictions (left) and measurements (right).
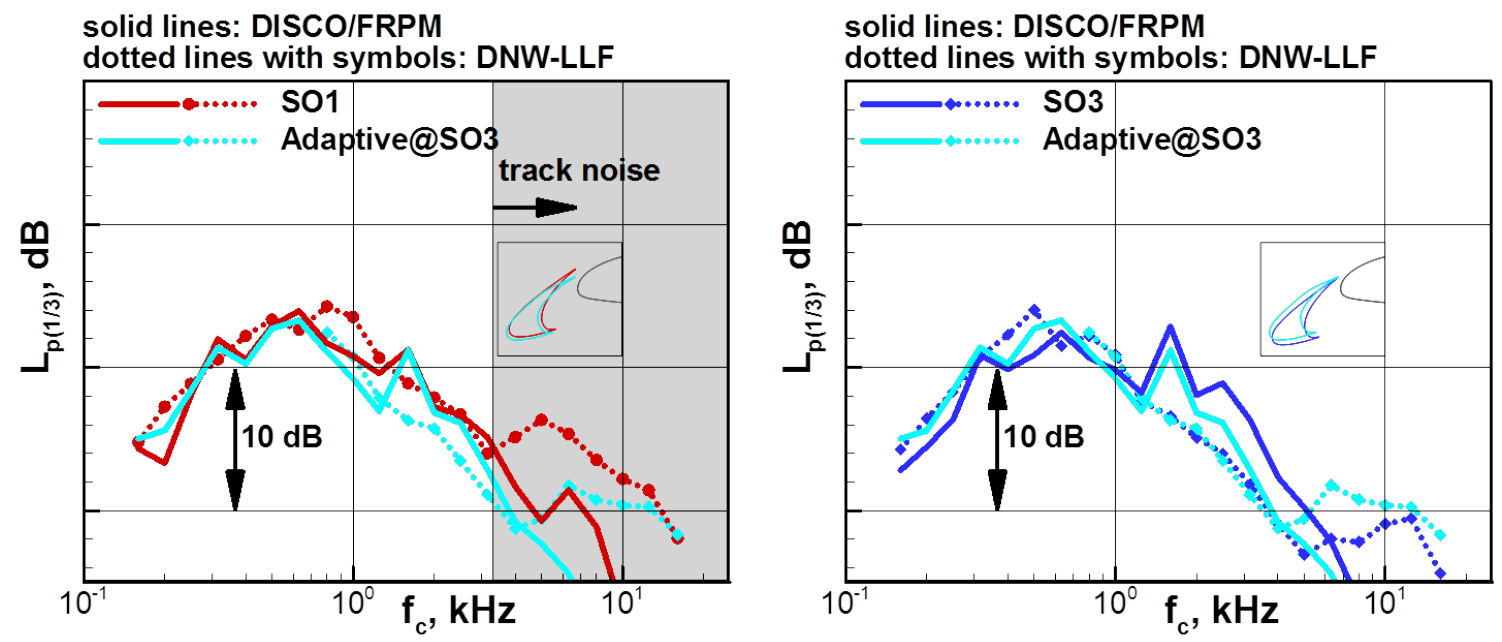

Figure 18. Comparison of predicted and measured trends, $\alpha=5^{\circ}, u_{\infty}=65 \mathrm{~m} / \mathrm{s}, \theta=90^{\circ}$. Left: effect of gap variation realized by adaptive slat (intermediate gap width). Right: shape effect of adaptive slat; same gap/overlap setting realized by conventional or adaptive slat, respectively. Data at $f<0.8 \mathrm{kHz}$ for the adaptive slat are omitted for clarity, cf. figure 14 . 
solid lines: PIANO/RPM

dotted lines with symbols: DNW-LLF
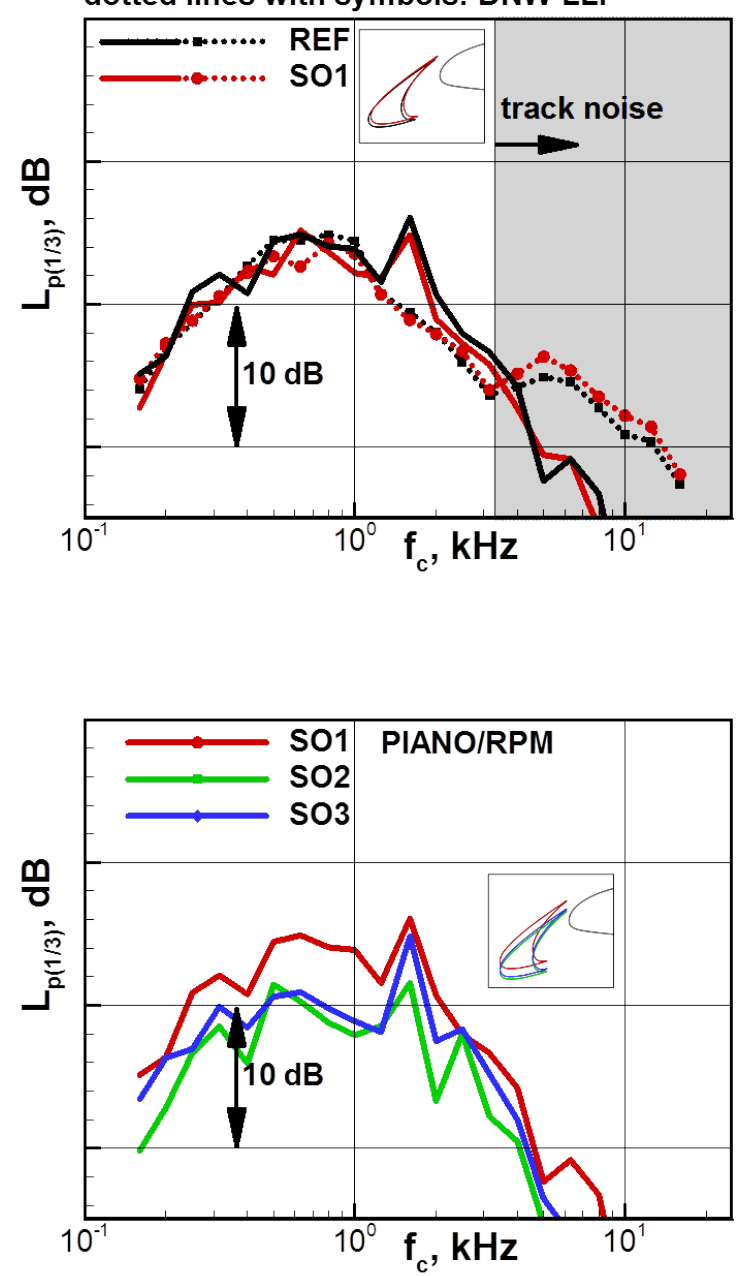

solid lines: PIANO/RPM

dotted lines with symbols: DNW-LLF

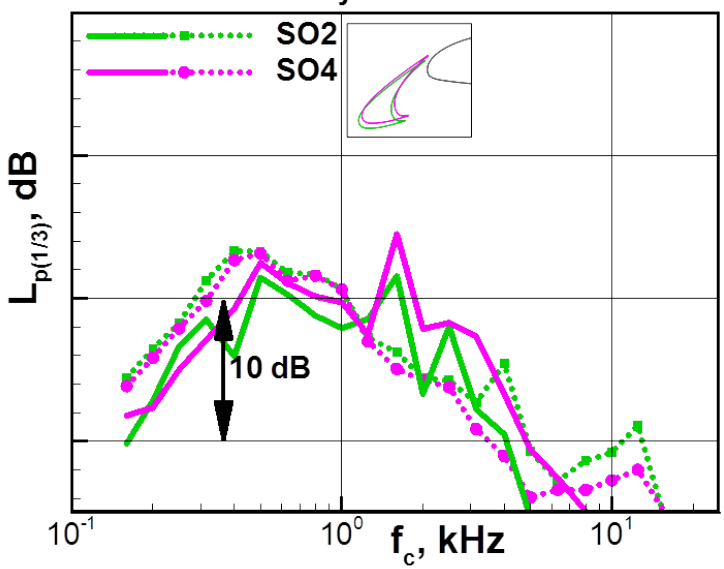

solid lines: PIANO/RPM

dotted lines with symbols: DNW-LLF

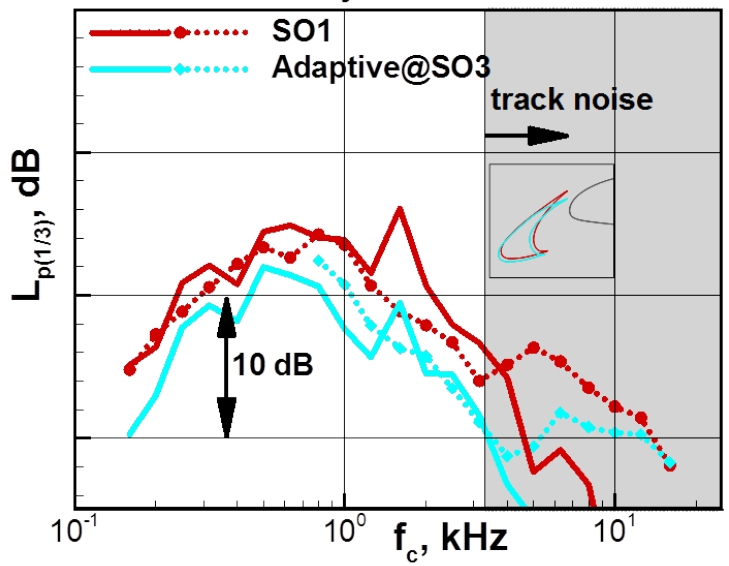

Figure 19. Trend predictions derived on earlier numerical simulations based on the PIANO-RPM framework. Same test cases as in the above figures. 


\section{Summary, conclusions and outlook}

The current contribution summarizes the slat noise research activity within work package WP4.2 (Wing Systems) of the EC co-financed project OPENAIR (Optimization for low environmental noise impact aircraft) conducted by the partners Airbus and DLR. It involves both experimental and numerical studies at the largescale (1:3.3) swept high-lift wing model F15LS. Experiments were performed in the Large Low-speed Facility DNW-LLF during the first test entry of this model, accomplished in the framework of a joint measurement campaign of the projects FTEG and OPENAIR. The presented slat noise studies build upon the outcome of the forerunner project TIMPAN, where the noise differences attributed to slat setting variations were numerically predicted and experimentally validated at a fourfold smaller 1:13.2-scaled 2D high-lift system (F16 model). Major achievements of the large-scale approach were:

- Existing data bases for established slat noise reduction concepts were extended towards more realistic conditions. Particularly, large-scale swept slat noise verification and validation data were collected at a maximum Reynolds number of $R e=5.1 \times 10^{6}$.

- The DNW-LLF noise assessment confirmed former (small scale, 2D) noise reduction results from the forerunner EC projects TIMPAN and NACRE: (i) A 3-dB noise reduction at wing level was obtained for the best slat setting. Overall, a relatively broad optimum for low-noise slat settings was confirmed. The induced aerodynamic impact at the operative test angles of attack was found negligible. (ii) Up to $5 \mathrm{~dB}$ noise reduction at wing level was measured for the adaptive slat with sealed gap, being equivalent to a full removal of the slat noise source.

- Projection to flight provided a translation of these noise reduction results in terms of approach certification noise levels; a 0.5 -EPNdB reduction at aircraft level for the best slat setting (SO4) as well as for the adaptive slat (closed gap) is expected, when only the slat noise source is treated. This analysis includes a preliminary consideration of integration effects.

- FEM analysis served to specify technically feasible adaptive slat profile bending, accounting for conventional actuator specifications, feasible skin materials and critical aerodynamic loads determined by the flight envelope.

- DLR's CAA-codes PIANO and DISCO were both presented to be applicable tools for slat noise prediction and corresponding low-noise design. The new discontinuous Galerkin (DG) CAA code DISCO was applied to selected 2D slat setting variants. DISCO works on an unstructured grid based on triangular elements. Since un-structured meshes are more conveniently applicable to complex geometries than structured ones, this approach includes the ability to test several settings with short mesh generation time as it is desirable for industrial applications. The changeover to unstructured grids is seen as an important achievement to cope with future demands.

Future needs involve a more detailed 3D assessment (including weight penalties) of feasible adaptive slat concepts or alternative system solutions. Corresponding studies are ongoing. An aerodynamical assessment of the adaptive slat within the operational linear region of the lift polar is expected from smaller-scale experiments. Further refinements are necessary to improve the quality of the shown comparisons in this report. These include: (i) the acoustic data analysis and post-processing, supplemented by the results of newer tests at the F15LS model, focusing at a detailed understanding of observed tonal phenomena, and (ii) the definitions of CAA 'best-practice' settings to further minimize the observed scatter in derived prediction results, given the relatively small noise reduction differences to be resolved for acoustic optimization purposes.

\section{Acknowledgments}

This study was part of the EC co-financed project OPENAIR under FP7 grant agreement No. 234313.

\section{References}

\footnotetext{
${ }^{1} \mathrm{M}$. Bauer, J. Dierke, and R. Ewert. Application of a Discontinuous Galerkin Method to Discretize Acoustic Perturbation Equations. AIAA Journal, 49(5), 2011.
} 
${ }^{2}$ M. Bauer and R. Ewert. Slat Noise Prediction using Discontinuous Galerkin Method and Stochastic Turbulent Sound Source. AIAA Paper 2010-3832, June 2010.

${ }^{3}$ D. Boenke, R. Ewert, and J. Delfs. Validation of Hybrid Numerical Methods for Aeroacoustic Simulations in an Industrial Environment. Conference Procs., CEAS/XNOISE Workshop for Aircraft Noise Reduction by Flow Control and Active/Adaptive Techniques, 25-26 September 2014, Vilnius, Lithuania, 2014.

${ }^{4}$ D. Cadot-Burillet, P. Lempereur, A. Rossetti, D. Collin, and A. Kempton. Technology Evaluator: A Global Way of Assessing Low Noise Technologies. AIAA Paper 2007-3669, May 2007.

${ }^{5}$ J. Delfs. Strömungsgeräusche - Beschreibung und Minderung mittels numerischer und experimenteller Simulation. Fortschritte der Akustik - DAGA 11, 1:21-32, 21-24 March 2011. CD Conference Paper, 34. Jahrestagung für Akustik.

${ }^{6}$ J. Delfs. Technical report, submitted to EU Proposal "OPENAIR" 28 Nov. 2007, contained in final "OPENAIR" proposal, submitted 7 May 2008 .

${ }^{7}$ J.W. Delfs, M. Bauer R. Ewert, H.A. Grogger, M. Lummer, and T.G.W.A. Lauke. Numerical Simulation of Aerodynamic Noise with DLR's Aeroacoustic Code PIANO. DLR internal report PIANO manual verion 5.2, 2007.

${ }^{8}$ W. Dobrzynski. Almost 40 Years of Airframe Noise Research-What Did we Achieve? Journal of Aircraft, 47(2):353-367, April 2010. 2001.

${ }^{9}$ W. Dobrzynski and M. Pott-Pollenske. Slat Noise Studies for Farfield Noise Prediction. AIAA Paper 2001-2158, May

${ }^{10}$ R. Ewert. RPM-The Fast Random Particle-Mesh Method to Realize Unsteady Turbulent Sound Sources and Velocity Fields for CAA Applications. Technical report, 2007.

${ }^{11}$ R. Ewert. Broadband Slat Noise Prediction Based on CAA and Stochasic Sound Sources from a Fast Random ParticleMesh (RPM) Method. Computers \& Fluids, 37:369-387, 2008.

${ }^{12}$ R. Ewert, C. Appel, J. Dierke, and M. Herr. RANS/CAA Based Prediction of NACA 0012 Broadband Trailing Edge Noise and Experimental Validation. AIAA Paper 2009-3269, AIAA-Paper, May 2009.

${ }^{13}$ R. Ewert, J. Dierke, M. Pott-Pollenske, C. Appel, R. Emunds, and M. Sutcliffe. CAA-RPM Prediction and Validation of Slat Setting Influence on Broadband High-Lift Noise Generation. AIAA Paper 2010-3833, June 2010.

${ }^{14}$ R. Ewert, J. Dierke, J. Siebert, A. Neifeld, C. Appel, M. Siefert, and O. Kornow. CAA Broadband Noise Prediction for Aeroacoustic Design. Journal of Sound and Vibration, 330:4139-4160, 2011.

${ }^{15}$ R. Ewert and R. Emunds. CAA Slat Noise Studies Applying Stochastic Sound Sources Based On Solenoidal Digital Filters. AIAA Paper 2005-2862, 2005.

${ }^{16}$ R. Ewert and W. Schröder. Acoustic Perturbation Equations Based on Flow Decomposition via Source Filtering. J. Comput. Phys., 188:365-398, 2003.

${ }^{17}$ Y. Guo. Slat Noise Modelling and Prediction. Journal of Sound and Vibration, 331:3567-3586, 2012.

${ }^{18}$ Y. Guo. Aircraft Slat Noise Modeling and Prediction. AIAA Paper 2010-3837, June 2010.

${ }^{19}$ M. Kintscher, O. Heintze S. Geier, and A. Rudenko. Adaptive Slat. European Patent Application EP-2505493 A1, registered 22 March 2012.

${ }^{20}$ L. Liu, X. Li, and F.Q. Hu. Non-Uniform Time-Step Runge-Kutta Discontinuous Galerkin Method for Computational Aeroacoustics. J. Comput. Phys., 229:6874-6897, 2010.

${ }^{21}$ M. Pott-Pollenske, J. Alvarez-Gonzalez, and W. Dobrzynski. Effect of Slat Gap on Farfield Radiated Noise and Correlation with Local Flow Characteristics. AIAA Paper 2003-3228, May 2003.

${ }^{22}$ M. Pott-Pollenske, J. Delfs, and J. Reichenberger. A Testbed for Large Scale and High Reynolds Number Airframe Noise Research. AIAA Paper 2013-2260, May 2013.

${ }^{23}$ R. J. Purser, W.-S. Wu, D. F. Parrish, and N. M. Roberts. Numerical Aspects of the Application of Recursive Filters to Variational Statistical Analysis. Part I: Spatially Homogeneous and Isotropic Gaussian Covariances. Monthly Weather Review, 131:1524-1535, 2003.

${ }^{24}$ R. J. Purser, W.-S. Wu, D. F. Parrish, and N. M. Roberts. Numerical Aspects of the Application of Recursive Filters to Variational Statistical Analysis. Part II: Spatially Inhomogeneous and Ansiotropic General Covariances. Monthly Weather Review, 131:1536-1548, 2003.

${ }^{25}$ N. Reiche, M. Lummer, and R. Ewert. Towards High-Lift Noise from Fast Multipole BEM with Anisotropic Synthetic Turbulence Sources. AIAA Paper 2014-2672, 2015.

${ }^{26}$ M. Siefert and R. Ewert. Sweeping Sound Generation in Jets Realized with a Random Particle-Mesh Method. AIAA Paper 2009-3369, 2009.

${ }^{27}$ P. Sijtsma. CLEAN Based on Spatial Source Coherence. AIAA Paper 2007-3436, May 2007.

${ }^{28}$ C. Tam and J. Webb. Dispersion-Relation-Preserving Finite Difference Schemes for Computational Acoustics. J. Comp. Phys., 107:262-281, 1993.

${ }^{29}$ I. T. Young and L. J. van Vliet. Recursive Implementation of the Gaussian Filter. Signal Processing, 44:139-151, 1995. 